\title{
Morphoelastic Rods III: Differential Growth and Curvature Generation in Elastic Filaments
}

\author{
Derek E. Moulton*, Thomas Lessinnes ${ }^{\dagger}$, Alain Goriely* \\ * Mathematical Institute, University of Oxford \\ ${ }^{\dagger}$ Polytechnique, Université Libre de Bruxelles
}

May 5, 2020

- Abstract In many growing filamentary structures such as neurons, roots, and stems, the intrinsic shapes and material response is produced by differential growth of the tissue. Therefore, a key problem is to link the growth field at the microscopic level to the macroscopic shape and properties of the filaments. Here, starting with a morphoelastic tubular structure and assuming a local growth law on the growth tensor, we use dimensional reduction to obtain the overall curvature, torsion, and material parameters of a growing filament. Various examples of curvature and torsion generation are given and the impact of residual stress on the generation of curvature is demonstrated.

\section{Introduction}

The shape of many biological filaments is dictated by differential growth. Consider the simple situation of an initially straight cylinder lying horizontally and made of a biological material capable of growing. Assume that points on the bottom half of the cross section grow faster along the axis than points on the top part. This difference in growth will create stresses that are partially relieved by modifying the shape of the central axis. In this simple case, the cylinder will curl up. Many such examples are provided by the study of plants. For instance, it is well known that inclining a plant will produce a gravitropic response where part of the stem will grow faster as to recover ascension against gravity by locally creating curvature $[1,2,3]$. Similarly, for a twining vine to grow helically around a pole, different points in the section normal to the axis must grow at different rates $[4,5]$ in order to generate both torsion and curvature. Another interesting case of differential growth is found in the main trunk of some trees that grow straight but twisted [6]. It is believed that this twist comes from a balance between growth and anisotropic response of the wood similar to the response found in some fungi such as phycomyces sporangium [7].

A natural question is then: If the local growth field of a filamentary structure is known at each point, what is the curvature, torsion, and twist of its centreline? And, What are its stiffness properties in response to bending, twisting, and stretching? We can look at this question as a multiscale problem relating the microscopic knowledge of growth at the tissue and cell levels viewed as a three-dimensional continuum to the macroscopic shape of the filament viewed as a simpler onedimensional elastic structure [8,9]. The main advantage is that a morphoelastic rod $[10,11]$ is a greatly simplified model that can easily be studied, even under complex deformations, to test various hypotheses on developmental patterns and the response of biological organisms to external stimuli $[12,13]$. The observable shape of the filamentary structure is linked to its fundamental growth processes. 
In the previous installments of this work $[10,11]$, we assumed that growth was only axial and the curvatures were known. One of the important ingredients entering any rod theory is the curvature. In growing systems, the curvatures are typically generated by a differential growth process and a fundamental problem is to link explicitly differential growth to the generation of curvatures. This is the main goal of this paper. Here, we treat the microscopic filament as a morphoelastic solid. It is a hyperelastic continuum that can grow and remodel and support stresses $[14,15,16]$. The macroscopic filament is then viewed as a morphoelastic rod: a one-dimensional elastic rod that can grow and remodel $[11,10]$. For both objects, we use the theory of morphoelasticity, which models growth through a multiplicative decomposition of the deformation gradient; we mostly follow the notations and definitions from [16].

Our approach consists in reducing the three-dimensional energy of a growing tubular structure to a one-dimensional energy of an elastic rod by first minimizing the energy over possible deformations in the cross section and, second, by averaging the resulting deformations over the tube's cross sections using the approach developed in [17] (see also $[18,19]$ for similar earlier theories). The emphasis here is on the explicit construction of the curvatures and the relationship between microscopic growth fields and macroscopic shape, noting that much of the resulting theory can be obtained through the idea of Gamma convergence as has been done by multiple authors [20, 21, 22] with results similar to [23] (see also [24] for similar ideas applied to plate theory). We consider two cases: first, we assume that the growth tensor is close to the identity with a perturbation depending on the small cross-sectional lengthscale. In this case, intrinsic curvatures can be obtained systematically and we recover results from these studies. Second, we look at the case where the growth tensor is the product of a finite deformation preserving the cylindrical symmetry and a near-identity deformation inducing curvatures.

Inspired by the world of plants, we apply these ideas to simple scenarios of differential growth in the section leading to changes in curvature and the formation of helical structures. Nevertheless, these methods are universal for anelastic variations within a tubular section that can be described by the multiplicative decomposition of the deformation gradient (thermo-elasticity [25], elastoplasticity [26], active processes [27], dislocations [28] and so on [29]). The paper is organized as follows: In Section 2, we discuss the general step-by-step algorithm needed to obtain curvatures and stiffnesses from the knowledge of the growth tensor and hyperelastic strain-energy density. Then, we consider several important cases where analytic progress can be made and general formulas can be obtained. First, we demonstrate the generation of curvature and torsion via incremental axial growth in Section 3. The effect of finite growth and residual stress is then developed in Section 4.

\section{General set-up}

The dimensional reduction starts by assuming that the filament is a three-dimensional tubular body that is allowed to grow. The initial structure is stress-free and growth is defined at every point as a local change of a volume element. As a result, the body will develop residual elastic energy [30]. This build up of elastic energy due to growth is reduced by small deformations within the section (reactive strains) and by deformation of the centreline (curvatures). Explicitly, the procedure is decomposed into the following steps.

Step 1: The growth tensor. We define within a cylindrical structure a growth tensor, characterizing at each point the local change of shape of a volume element by the addition, removal, or redistribution of mass.

Step 2: Define a deformation gradient. We assume that this filamentary structure can be deformed both by deformations within the section and deformation of the centreline. This 
step naturally introduces a small parameter $\varepsilon$ defined as the ratio of the typical size of the section $R$ to the length $L$ of the rod: $\varepsilon=R / L \ll 1$.

Step 3: Compute the energy. Given the deformation gradient, the energy density can be expanded asymptotically in powers of $\varepsilon$.

Step 4: Minimize the energy over the section. To each order, we can minimize the energy integrated over the section with respect to deformations within the section. These are the reactive strains developed within the rod [31].

Step 5: Average over the section. Once the reactive strains are known, the energy density is averaged over the cross section and an expression is obtained for the energy in terms of the curvatures that can be identified with the energy of a rod.

\subsection{Step 1: The growth tensor}

We consider an initial tubular configuration $\mathcal{B}_{0} \subset \mathbb{R}^{3}$ with material points $(X, Y, Z) \in \mathcal{B}_{0}$ as shown in Figure 1. In this tubular description $\mathcal{B}_{0}$ can be decomposed as the product $[0, L] \times \mathcal{S}$ of a segment of the $Z$-axis between 0 and $L$ and a family of cross-sections $\mathcal{S}_{Z}$ whose centroids are on the $Z$-axis and oriented so that

$$
\int_{\mathcal{S}_{Z}} X \mathrm{~d} X \mathrm{~d} Y=\int_{\mathcal{S}_{Z}} Y \mathrm{~d} X \mathrm{~d} Y=\int_{\mathcal{S}_{Z}} X Y \mathrm{~d} X \mathrm{~d} Y=0
$$

We note that in the case where $\mathcal{S}_{Z}$ is independent of $Z$, one can always choose a system of coordinates so that this assumption is satisfied. The general case is a bit more involved and can be carried out at the expense of extra terms in the final result. We assume that the typical length scale of each section is of order $\mathcal{O}(\varepsilon)$ and that each cross section is a slowly varying function of the arc length $Z$, so that on short scales, the tubular structure is cylindrical.

We use the framework of morphoelasticity [16] and model growth through a tensor $\mathbf{G}$. We consider the deformation $\chi(\mathbf{X})$ from the initial configuration $\mathcal{B}_{0}$ to the current configuration $\mathcal{B}$. The associated deformation gradient is

$$
\mathbf{F}=\operatorname{Grad} \chi
$$

where the gradient is taken with respect to the $\mathbf{X}$ coordinates. Following the fundamental assumption of morphoelasticity [32], we assume that this deformation gradient can be decomposed into a growth part $\mathbf{G}$ and an elastic part $\mathbf{A}$ so that $\mathbf{F}=\mathbf{A G}$. The elasticity tensor $\mathbf{A}$ describes the elastic stretches that are governed by a strain-energy density function $W=W(\mathbf{A})$. The growth tensor $\mathbf{G}$ is considered to be an input in our problem and is given by an invertible tensor with strictly positive determinant

$$
\mathbf{G}=G_{i j} \mathbf{e}_{i} \otimes \mathbf{e}_{j}, \quad i, j \in\{x, y, z\},
$$

where $\left(\mathbf{e}_{1}, \mathbf{e}_{2}, \mathbf{e}_{3}\right)$ are the usual unit Cartesian basis vectors along $\mathbf{X}=(X, Y, Z)$ and, in general, $G_{i j}=G_{i j}(X, Y, Z)$.

In a biological context, this growth tensor expresses the local change or rearrangement of cells due to growth or remodeling. For instance if cells are assumed to only grow by elongating in the $Z$ direction, then the growth tensor will be of the form $\mathbf{G}=\operatorname{diag}\left(1,1, \gamma_{Z}\right)$ with $\gamma_{Z}>1$ describing the change in length due to growth.

Here we consider growth tensors that are themselves a product

$$
\mathbf{G}=\left(\mathbf{1}+\mathbf{G}_{1}(\mathbf{X})\right) \mathbf{G}_{0}(\mathbf{X}),
$$




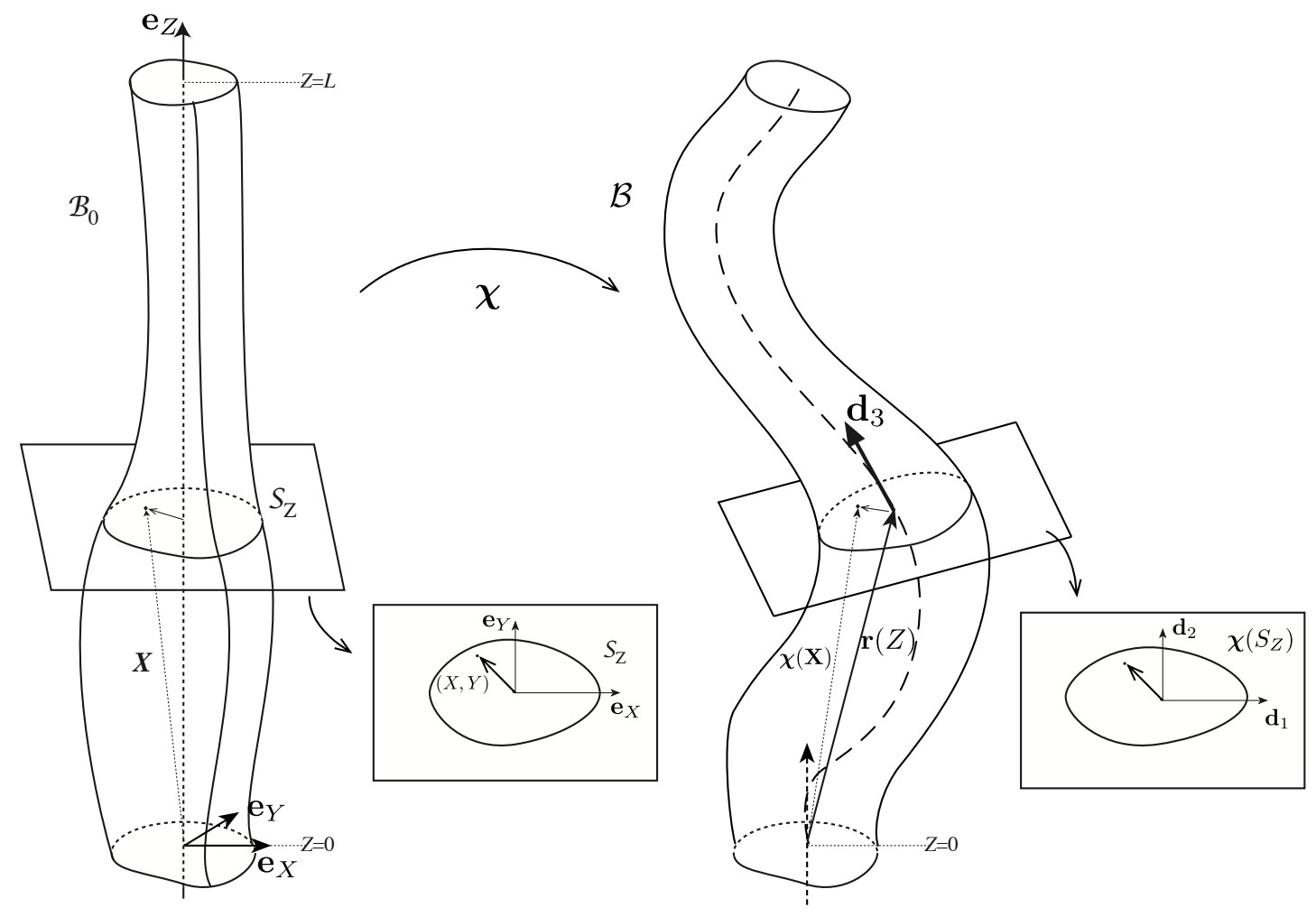

Figure 1: In its undeformed configuration $\mathcal{B}_{0}$, a tubular body has a straight centroid axis and arbitrary cross-sections. Following growth and elastic relaxation, the body is described in configuration $\mathcal{B}$ by a central curve $\mathbf{r}(Z)$ equipped with a local orthonormal frame $\left\{\mathbf{d}_{1}, \mathbf{d}_{2}, \mathbf{d}_{3}\right\}$ (see Appendix A for details).

of a finite growth deformation $\mathbf{G}_{0}$ that does not induce any curvature, and an incremental deformation $\left(\mathbf{1}+\mathbf{G}_{1}\right)$ that may induce curvature and torsion. The tensor $\mathbf{G}_{1}$ must be taken to be small in a way that will be defined shortly.

We consider a particular family of possible deformations, mapping a straight tubular structure to a filament in space $\mathcal{B}$ with centreline $\mathbf{r}(Z)$ (see Fig. 1). This centreline is the image of a segment of the $Z$-axis defining the centreline of the initial configuration. As explained in details in Appendix A, from this centreline, we define a local director basis $\left(\mathbf{d}_{1}(Z), \mathbf{d}_{2}(Z), \mathbf{d}_{3}(Z)\right)$ where $\mathbf{r}^{\prime}(Z)=\zeta \mathbf{d}_{3}$ and $\zeta$ is the axial extension and ()$^{\prime}$ denotes derivatives with respect to the material coordinate $Z$. From the director basis, we define the Darboux curvature vector $\mathbf{u}=\mathbf{u}_{1} \mathbf{d}_{1}+\mathbf{u}_{2} \mathbf{d}_{2}+\mathbf{u}_{3} \mathbf{d}_{3}$. This vector describes the evolution of the director basis along the filament, satisfiying

$$
\mathbf{d}_{i}^{\prime}(Z)=\zeta \mathbf{u} \times \mathbf{d}_{i} .
$$

The mapping $\chi: \mathcal{B}_{0} \rightarrow \mathcal{B}$ is then written

$$
\chi(\mathbf{X})=\mathbf{r}(Z)+\sum_{i=1}^{3} \rho_{i}(X, Y, Z) \mathbf{d}_{i}(Z)
$$

where the $\rho_{i}$ are functions to be determined that describe the local deformation of the section; these satisfy $\rho_{i}(0,0, Z)=0$ so that the $Z$-axis maps to the centerline $\mathbf{r}(Z)$. This particular form (6) expresses the deformation of a tubular body in terms of its centreline and director basis, and is 
key to the development. Before we compute the gradient, we take advantage of the small parameter $\varepsilon$ and introduce the following scaling

$$
X=\varepsilon x, \quad Y=\varepsilon y, \quad Z=Z, \quad \rho_{i}=\varepsilon \alpha_{i} .
$$

In these new variables, and using the notation $\alpha_{1 x}=\partial_{x} \alpha_{1}$ and so on, the deformation gradient reads:

$$
\mathbf{F}=F_{i j} \mathbf{d}_{i} \otimes \mathbf{e}_{j}=\left[\begin{array}{ccc}
\alpha_{1 x} & \alpha_{1 y} & (1+\varepsilon \xi) \varepsilon\left(\mathbf{u}_{2} \alpha_{3}-\mathbf{u}_{3} \alpha_{2}\right) \\
\alpha_{2 x} & \alpha_{2 y} & (1+\varepsilon \xi) \varepsilon\left(\mathbf{u}_{3} \alpha_{1}-\mathbf{u}_{1} \alpha_{3}\right) \\
\alpha_{3 x} & \alpha_{3 y} & (1+\varepsilon \xi)\left(1+\varepsilon\left(\mathbf{u}_{1} \alpha_{2}-\mathbf{u}_{2} \alpha_{1}\right)\right)
\end{array}\right]
$$

where $\zeta=1+\varepsilon \xi$, so that $\xi$ captures the (small) axial strain.

\subsection{Step 3: The energy density}

We assume that the growing material is hyperelastic with strain-energy density $W=W(\mathbf{A})$. The material may be be either compressible or incompressible and the equilibrium problem is to minimize the energy

$$
\mathcal{E}=\int_{\mathcal{B}_{0}}(W(\mathbf{A})-p(J-1)) \operatorname{det} \mathbf{G} \mathrm{d} V
$$

over all possible deformations considered above. Here, $J=\operatorname{det} \mathbf{A}$ and $p$ is a Lagrange multiplier to be determined in the minimization process if the material is incompressible. The factor det $\mathbf{G}$ appears since the integral is written in the initial pre-grown configuration, while the energy density is an energy per reference grown volume. In general, we define the potential

$$
V=(W(\mathbf{A})-p(J-1)) \operatorname{det} \mathbf{G},
$$

where $p=0$ in the compressible case.

Using the decomposition of the deformation gradient $\mathbf{F}=\mathbf{A} \mathbf{G}$ and the invertibility of $\mathbf{G}$, we can express the potential as

$$
V=V\left(\mathbf{F G}^{-1}, \mathbf{G}\right) .
$$

Then, the energy functional can be written

$$
\mathcal{E}=\varepsilon^{2} \int_{0}^{L} \mathcal{F}(Z) \mathrm{d} Z, \quad \mathcal{F}(Z)=\int_{\tilde{\mathcal{S}}} V \mathrm{~d} x \mathrm{~d} y,
$$

where $\tilde{\mathcal{S}}$ denotes the scaled cross section (i.e. $(X, Y) \in \mathcal{S}$ implies that $(x, y) \in \tilde{\mathcal{S}}$ ).

\subsection{Step 4: Minimization over the section}

The problem now is to find the functions $\boldsymbol{\alpha}=\left(\alpha_{1}, \alpha_{2}, \alpha_{3}\right)$ and $p$ that minimize the energy over each section. The main idea is to expand these variables in the small parameter $\varepsilon$ :

$$
\begin{aligned}
& \boldsymbol{\alpha}=\boldsymbol{\alpha}^{(0)}+\varepsilon \boldsymbol{\alpha}^{(1)}+\ldots \\
& p=p^{(0)}+\varepsilon p^{(1)}+\ldots
\end{aligned}
$$

For the incremental growth to have an effect on the energy at order $\mathcal{O}\left(\varepsilon^{2}\right)$ (and not at lower order), it must scale as $\mathbf{G}_{1}=\varepsilon \mathbf{g}_{1}(\mathbf{x})$, so that

$$
\mathbf{G}=\left(\mathbf{1}+\varepsilon \mathbf{g}_{1}(\mathbf{x})\right) \mathbf{G}_{0} .
$$


Then, for $\mathbf{F}$ given in (8), the strain-energy density can be expanded in $\varepsilon$

$$
V=V_{0}\left(\boldsymbol{\alpha}^{(0)}, p^{(0)}\right)+\varepsilon V_{1}\left(\boldsymbol{\alpha}^{(0)}, \boldsymbol{\alpha}^{(1)}, p^{(0)}, p^{(1)}\right)+\varepsilon^{2} V_{2}\left(\boldsymbol{\alpha}^{(0)}, \boldsymbol{\alpha}^{(1)}, \boldsymbol{\alpha}^{(2)}, p^{(0)}, p^{(1)}, p^{(2)}\right)+\ldots
$$

from which we define a sequence of minimization problems for the functionals

$$
\mathcal{F}_{i}\left[\boldsymbol{\alpha}^{(0)}, \ldots, \boldsymbol{\alpha}^{(k)} ; p^{(0)}, \ldots, p^{(k)}\right]=\int_{\tilde{\mathcal{S}}} V_{i} \mathrm{~d} x \mathrm{~d} y, \quad i=0,1,2, \ldots
$$

For a cross-section $\tilde{\mathcal{S}}$, the associated Euler-Lagrange equations for these problems take the form

$$
\begin{array}{ll}
\left(\frac{\partial}{\partial x}, \frac{\partial}{\partial y}\right) \cdot\left(\frac{\partial V_{i}}{\partial \alpha_{j x}^{(k)}}, \frac{\partial V_{i}}{\partial \alpha_{j y}^{(k)}}\right)-\frac{\partial V_{i}}{\partial \alpha_{j}^{(k)}}=0, & j=1,2,3 \\
\left(\frac{\partial}{\partial x}, \frac{\partial}{\partial y}\right) \cdot\left(\frac{\partial V_{i}}{\partial p_{x}^{(k)}}, \frac{\partial V_{i}}{\partial p_{y}^{(k)}}\right)-\frac{\partial V_{i}}{\partial p^{(k)}}=0, & (x, y) \in \tilde{\mathcal{S}}
\end{array}
$$

with the natural boundary conditions

$$
\begin{aligned}
& \left(\frac{\partial V_{i}}{\partial \alpha_{j x}^{(k)}}, \frac{\partial V_{i}}{\partial \alpha_{j y}^{(k)}}\right) \cdot \mathbf{n}=0, \quad j=1,2,3 \\
& \left(\frac{\partial V_{i}}{\partial p_{x}^{(k)}}, \frac{\partial V_{i}}{\partial p_{y}^{(k)}}\right) \cdot \mathbf{n}=0, \quad(x, y) \in \partial \tilde{\mathcal{S}}
\end{aligned}
$$

where $\mathbf{n}$ is the unit external normal vector at the boundaries $\partial \tilde{\mathcal{S}}$ of $\tilde{\mathcal{S}}$. At each order $\varepsilon^{i}$, the choice of $k \leq i$, and thus the functions $\boldsymbol{\alpha}^{(k)}, p^{(k)}$ to be satisfied by the Euler-Lagrange equations, depends on the solution at previous order. For instance, we will see that for $i=1$ the equations are automatically satisfied, hence $\boldsymbol{\alpha}^{(1)}, p^{(1)}$ is only solved at order $\varepsilon^{2}$. By construction, the PDE system (18)-(21) is linear in $\boldsymbol{\alpha}^{(k)}, p^{(k)}$ and can in principle be solved for a given growth tensor $\mathbf{G}$ and strain-energy density $W=W(\mathbf{A})$.

\subsection{Step 5: Averaging over a section}

Once the solution for $\boldsymbol{\alpha}, p$ is known up to order $\varepsilon$, the energy takes the form

$$
\mathcal{E}=\varepsilon^{4} \int_{0}^{L} \mathrm{~d} Z \int_{\tilde{\mathcal{S}}} V_{2}\left(\boldsymbol{\alpha}^{(0)}, \boldsymbol{\alpha}^{(1)}, p^{(0)}, p^{(1)} ; \mathbf{u}_{1}, \mathbf{u}_{2}, \mathbf{u}_{3}, \xi\right) \mathrm{d} x \mathrm{~d} y+\mathcal{O}\left(\varepsilon^{5}\right)
$$

The elastic energy of the rod is then obtained by integrating $V_{2}$ over the cross sections. Having expanded the strain-energy density to $\mathcal{O}\left(\varepsilon^{2}\right)$, and since by construction $\boldsymbol{\alpha}^{(1)}$ and $p^{(1)}$ depend on the curvature $\mathbf{u}$ and extensional strain $\xi$, once the integration over $x$ and $y$ is performed, (22) has the form of an integral only along $Z$ and with quadratic dependence on the quantities $\left\{\mathrm{u}_{1}, \mathrm{u}_{2}, \mathrm{u}_{3}, \xi\right\}$. Thus, we have converted the energy to that of a Kirchhoff elastic rod with material variable $Z=S$. It is then easy to identify, by comparison with a classic rod energy, the intrinsic curvatures giving the unstressed shape of the rod and its stiffnesses. Rather than giving the most general form for these intrinsic curvatures, we start with simple examples to illustrate the procedure and build towards a general theory. 


\section{Incremental axial growth}

\subsection{Generating curvature through a growth gradient for a circular section}

We start with an elementary example capable of generating curvature but no torsion. We assume that the material is isotropic and compressible (hence $p=0$ ) and that the structure is initially cylindrical with length $L=1$ along the $Z$-axis and circular cross section $\mathcal{S}$ of radius $R=\varepsilon \ll 1$ and with uniform material properties (all elastic moduli are constant through the material). To set the orientation of the local frame, throughout this paper we identify the local basis in the undeformed state with the Cartesian basis $\left\{\mathbf{d}_{1}=\mathbf{e}_{X}, \mathbf{d}_{2}=\mathbf{e}_{Y}, \mathbf{d}_{3}=\mathbf{e}_{Z}\right\}$.

We assume no initial finite growth $\left(\mathbf{G}_{0}=\mathbf{1}\right)$ and take the incremental growth to be purely axial with a gradient along the $X$ axis:

$$
\mathbf{G}=\operatorname{diag}(1,1,1+\gamma X)=\operatorname{diag}(1,1,1+\varepsilon \gamma x) .
$$

A remarkable feature of the theory of rods is that since the strain-energy density is isotropic and its contribution in the expression for $V$ includes at most quadratic terms in the strains, we can use without loss of generality the quadratic approximation of $W$ :

$$
W=\frac{1}{2}\left[\mu\left(\operatorname{tr}\left(\mathbf{H} . \mathbf{H}^{T}\right)+\operatorname{tr}\left(\mathbf{H}^{2}\right)\right)+\lambda \operatorname{tr}(\mathbf{H})^{2}\right],
$$

where $\mathbf{H}=\mathbf{A}-\mathbf{1}$ and $\mu, \lambda$ are the Lamé parameters.

The expansion of

$$
V=W\left(\mathbf{F}\left(\mathbf{1}+\mathbf{G}_{1}\right)^{-1}\right) \operatorname{det}\left(\mathbf{1}+\mathbf{G}_{1}\right)=V_{0}+\varepsilon V_{1}+\varepsilon^{2} V_{2}+\ldots,
$$

proceeds by using $\mathbf{F}$ given by (8) and the expansion $\boldsymbol{\alpha}$ in (13). The Euler-Lagrange equations for $\mathcal{F}_{0}$ are automatically satisfied with the choice

$$
\boldsymbol{\alpha}^{(0)}=x \mathbf{d}_{1}+y \mathbf{d}_{2},
$$

and we can verify explicitly that both $V_{0}$ and $V_{1}$ vanish identically on this solution. The information about the curvatures are contained in the second-order term

$$
\begin{array}{r}
V_{2}=\frac{\mu}{2}\left(2 x \mathbf{u}_{3} \alpha_{3 y}^{(1)}-2 y \mathbf{u}_{3} \alpha_{3 x}^{(1)}+\left(\alpha_{1 y}^{(1)}\right)^{2}+2\left(\alpha_{2 y}^{(1)}\right)^{2}+\left(\alpha_{3 y}^{(1)}\right)^{2}+2\left(\alpha_{1 x}^{(1)}\right)^{2}+\left(\alpha_{2 x}^{(1)}\right)^{2}+\left(\alpha_{3 x}^{(1)}\right)^{2}+2 \alpha_{1 y}^{(1)} \alpha_{2 x}^{(1)}\right. \\
\left.+x^{2} \mathbf{u}_{3}^{2}+2\left(y \mathbf{u}_{1}-x \mathbf{u}_{2}-\gamma x+\xi\right)^{2}+y^{2} \mathbf{u}_{3}^{2}\right)+\frac{\lambda}{2}\left(\alpha_{2 y}^{(1)}+\alpha_{1 x}^{(1)}-x \mathbf{u}_{2}+y \mathbf{u}_{1}-\gamma x+\xi\right)^{2} .
\end{array}
$$

The Euler-Lagrange equations for this problem are

$$
\begin{aligned}
& \lambda\left(\alpha_{2 x y}^{(1)}+\alpha_{1 x x}^{(1)}-\gamma-\mathrm{u}_{2}\right)+\mu\left(\alpha_{1 y y}^{(1)}+\alpha_{2 x y}^{(1)}+2 \alpha_{1 x x}^{(1)}\right)=0, \\
& \lambda\left(\alpha_{2 y y}^{(1)}+\alpha_{1 x y}^{(1)}+\mathrm{u}_{1}\right)+\mu\left(2 \alpha_{2 y y}^{(1)}+\alpha_{1 x y}^{(1)}+\alpha_{2 y y}^{(1)}\right)=0, \\
& \alpha_{3 x x}^{(1)}+\alpha_{3 y y}^{(1)}=0 .
\end{aligned}
$$

We use the parameterization of the circle $(x, y)=(\cos \theta, \sin \theta), \theta \in[0,2 \pi]$ which gives $\mathbf{n}=$ $(\cos \theta, \sin \theta)$ and the natural boundary conditions

$$
\begin{aligned}
& \lambda \sin \theta\left(\alpha_{2 y}^{(1)}+\alpha_{1 x}^{(1)}+u-\gamma x+\xi\right)+\mu\left(2 \sin \theta \alpha_{2 y}^{(1)}+\cos \theta\left(\alpha_{1 y}^{(1)}+\alpha_{2 x}^{(1)}\right)\right)=0, \\
& \lambda \cos \theta\left(\alpha_{2 y}^{(1)}+\alpha_{1 x}^{(1)}+u-\gamma x+\xi\right)+\mu\left(\sin \theta\left(\alpha_{1 y}^{(1)}+\alpha_{2 x}^{(1)}\right)+2 \cos \theta \alpha_{1 x}^{(1)}\right)=0, \\
& \sin \theta \alpha_{3 y}^{(1)}+\cos \theta \alpha_{3 x}^{(1)}=0,
\end{aligned}
$$


where $u:=\left(\mathrm{u}_{1} \sin \theta-\mathrm{u}_{2} \cos \theta\right)$ and $\boldsymbol{\alpha}^{(1)}$ and its derivatives are evaluated on the unit circle. These equations have an exact solution given by

$$
\begin{aligned}
& \alpha_{1}^{(1)}=\frac{\lambda}{2(\mu+\lambda)}\left(-x \xi+\frac{1}{2}\left(x^{2}-y^{2}\right)\left(\gamma+\mathrm{u}_{2}\right)-x y \mathrm{u}_{1}\right), \\
& \alpha_{2}^{(1)}=\frac{\lambda}{2(\mu+\lambda)}\left(-y \xi+\frac{1}{2}\left(x^{2}-y^{2}\right) \mathrm{u}_{1}+x y\left(\gamma+\mathrm{u}_{2}\right)\right), \\
& \alpha_{3}^{(1)}=0 .
\end{aligned}
$$

We note that the conditions $\rho_{1}(0,0 . Z)=\rho_{2}(0,0 . Z)=0$ imply $\alpha_{1}^{(1)}(0,0 . Z)=\alpha_{2}^{(1)}(0,0 . Z)=0$ and, by substituting $x=y=0$, we can directly verify that our ansatz satisfies these conditions. With the solutions for $\boldsymbol{\alpha}^{(0)}$ and $\boldsymbol{\alpha}^{(1)}$ known explicitly, we can then explicitly integrate the strainenergy density $V_{2}\left(\boldsymbol{\alpha}^{(0)}, \boldsymbol{\alpha}^{(1)}, \mathrm{u}_{1}, \mathrm{u}_{2}, \mathrm{u}_{3}, \xi\right)$ over the cross-section. We find (using the original unscaled variables and dropping terms $\mathcal{O}\left(\varepsilon^{5}\right)$ and higher):

$$
\mathcal{E}=\frac{1}{2} \int_{0}^{L}\left(E \pi R^{2}(\zeta-1)^{2}+E \frac{\pi R^{4}}{4} \mathrm{u}_{1}^{2}+E \frac{\pi R^{4}}{4}\left(\mathrm{u}_{2}+\gamma\right)^{2}+E \frac{\pi R^{4}}{4(1+\nu)} \mathrm{u}_{3}^{2}\right) \mathrm{d} Z .
$$

Here we have used $\xi=(\zeta-1) / R$ and the standard conversion between Lamé parameters and the Young's modulus $E$ and Poisson ratio $\nu$ :

$$
\lambda=\frac{\nu E}{(1+\nu)(1-2 \nu)}, \quad \mu=\frac{E}{2(\nu+1)} .
$$

By direct comparison with the energy of an extensible elastic rod (see Appendix A for details),

$$
\mathcal{E}_{\text {rod }}=\frac{1}{2} \int_{0}^{L}\left(E A(\zeta-1)^{2}+E I_{1}\left(\mathrm{u}_{1}-\hat{\mathrm{u}}_{1}\right)^{2}+E I_{2}\left(\mathrm{u}_{2}-\hat{\mathrm{u}}_{2}\right)^{2}+\mu J\left(\mathrm{u}_{3}-\hat{\mathrm{u}}_{3}\right)^{2}\right) \mathrm{d} S,
$$

we conclude that the morphoelastic rod has acquired through a growth gradient along the $X$ direction an intrinsic curvature $\hat{u}_{2}=-\gamma$, as shown Fig. 2(a). We also see that the stiffnesses of the grown rod are identical to the stiffness of the original rod since for a circular cross-section we have

$$
A=\pi R^{2}, \quad I_{1}=I_{2}=\frac{\pi R^{4}}{4}, \quad J=2 I_{1}=\frac{\pi R^{4}}{2}
$$

and these dimensions have not changed in the grown rod.

\subsection{Generating curvature, twist and torsion through growth in an isotropic material}

Next, we generalize the previous case by considering an axial incremental growth function for a rod with section $\mathcal{S}$ and made out of an isotropic material. The computations in this case are very close to the previous case and we merely outline the main results. Again, we assume no initial finite growth $\left(\mathbf{G}_{0}=\mathbf{1}\right)$ and take an incremental growth of the form

$$
\mathbf{G}_{1}=\operatorname{diag}(0,0, \varepsilon g(x, y))=\operatorname{diag}(0,0, G(x / \varepsilon, Y / \varepsilon)) \text {. }
$$

Since we can always rescale the overall length of the rod, we assume, without loss of generality, that

$$
\int_{\tilde{\mathcal{S}}} g(x, y) \mathrm{d} x \mathrm{~d} y=0
$$


(a)

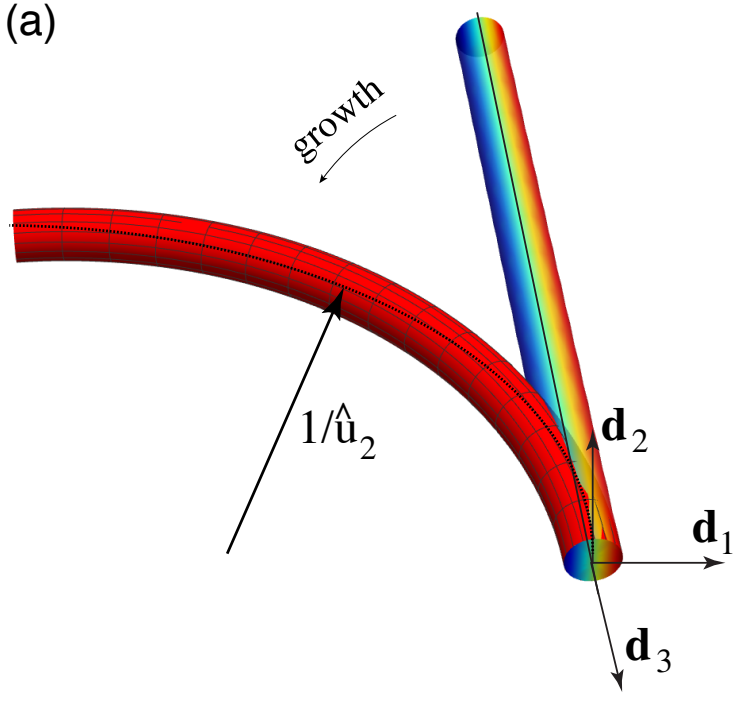

(b)

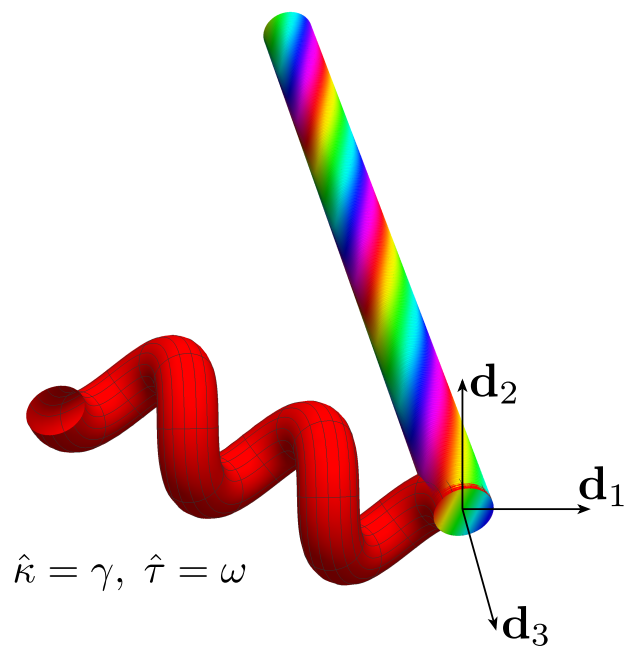

Figure 2: Generation of curvature and torsion by incremental axial growth in a cylindrical rod. In (a), a linear growth gradient $\gamma$ in the $\mathbf{e}_{X}$ direction generates a rod with intrinsic curvature $\gamma$. In (b), the linear growth gradient $\gamma$ twists along the $Z$ axis at rate $\omega$, thus generating both intrinsic curvature $\gamma$ and intrinsic torsion $\omega$.

which implies that there is no change of unstressed length due to growth.

The expansion of $V=V_{0}+\varepsilon^{2} V_{2}+\ldots$ proceeds as before and the resulting Euler-Lagrange equations associated with $V_{0}$ are again automatically satisfied by $\boldsymbol{\alpha}^{(0)}=x \mathbf{d}_{1}+y \mathbf{d}_{2}$, and $V_{1} \equiv 0$ again. The second-order term is

$$
\begin{aligned}
V_{2}= & \frac{\mu}{2}\left(2 x \mathbf{u}_{3} \alpha_{3 y}^{(1)}-2 y \mathbf{u}_{3} \alpha_{3 x}^{(1)}+\left(\alpha_{1 y}^{(1)}\right)^{2}+2\left(\alpha_{2 y}^{(1)}\right)^{2}+\left(\alpha_{3 y}^{(1)}\right)^{2}+2\left(\alpha_{1 x}^{(1)}\right)^{2}+\left(\alpha_{2 x}^{(1)}\right)^{2}+\left(\alpha_{3 x}^{(1)}\right)^{2}\right. \\
& \left.+2 \alpha_{1 y}^{(1)} \alpha_{2 x}^{(1)}+x^{2} \mathbf{u}_{3}^{2}+2\left(y \mathbf{u}_{1}-x \mathbf{u}_{2}-g+\xi\right)^{2}+y^{2} \mathbf{u}_{3}^{2}\right)+\frac{\lambda}{2}\left(\alpha_{2 y}^{(1)}+\alpha_{1 x}^{(1)}-x \mathbf{u}_{2}+y \mathbf{u}_{1}-g+\xi\right)^{2}
\end{aligned}
$$

A solution of the corresponding Euler-Lagrange equations is given by

$$
\begin{aligned}
& \alpha_{1}^{(1)}=\frac{\lambda}{2(\mu+\lambda)}\left(-x \xi+\frac{1}{2}\left(x^{2}-y^{2}\right) \mathrm{u}_{2}-x y \mathrm{u}_{1}+2 f_{1}\right), \\
& \alpha_{2}^{(1)}=\frac{\lambda}{2(\mu+\lambda)}\left(-y \xi+\frac{1}{2}\left(x^{2}-y^{2}\right) \mathrm{u}_{1}+x y \mathrm{u}_{2}+2 f_{2}\right), \\
& \alpha_{3}^{(1)}=\mathrm{u}_{3} \phi .
\end{aligned}
$$

Here, $\phi$ is the warping function of classical rod mechanics [16, p. 111]. It is a solution of

$$
\begin{aligned}
& \Delta \phi=0, \quad(x, y) \in \tilde{\mathcal{S}} \\
& \mathbf{n} \cdot\left(\phi_{x}, \phi_{y}\right)=\mathbf{n} \cdot(y,-x), \quad(x, y) \in \partial \tilde{\mathcal{S}},
\end{aligned}
$$

where $\mathbf{n}$ is a unit outward normal vector to the boundary $\partial \tilde{\mathcal{S}}$. The functions $f_{1,2}$ are solutions of the linear system

$$
\begin{aligned}
& \lambda\left(f_{2 x y}+f_{1 x x}-2 g_{x}\right)+\mu\left(f_{1 y y}+f_{2 x y}+2 f_{1 x x}-2 g_{x}\right)=0, \\
& \lambda\left(f_{2 y y}+f_{1 x y}-2 g_{y}\right)+\mu\left(f_{2 y y}+f_{1 x y}+f_{2 x x}-2 g_{y}\right)=0
\end{aligned}
$$


with boundary conditions

$$
\begin{aligned}
& \left(\mu\left(f_{1 y}+f_{2 x}\right), \lambda f_{2 y}+(\lambda+2 \nu) f_{1 x}+2(\lambda+\nu) g\right) \cdot \mathbf{n}=0, \\
& \left.\left(\lambda f_{1 x}-(\lambda+2 \nu) f_{2 y}-2(\lambda+\nu) g\right), \mu\left(f_{1 y}+f_{2 x}\right)\right) \cdot \mathbf{n}=0 .
\end{aligned}
$$

An explicit solution for the extraneous functions $f_{1}, f_{2}$ is given in Appendix B. However, we will see that their particular forms are not important as these two functions do not enter the expression of the curvatures. It is instructive to observe that the cross-sectional geometry and the form of incremental growth, $g(x, y)$, only impact the extraneous functions, while the terms in the $\alpha_{i}^{(1)}$ that multiply the rod's strain components $\left\{\mathrm{u}_{1}, \mathrm{u}_{2}, \mathrm{u}_{3}, \xi\right\}$ are completely generic in that they do not depend on cross-sectional geometry nor on the form of incremental growth. The dependence on the cross-section and the growth function only enters through the form of the energy density at second order. Indeed, evaluated on the solutions for $\boldsymbol{\alpha}^{(0)}$ and $\boldsymbol{\alpha}^{(1)}$, the energy density reads

$$
\begin{aligned}
& V_{2}=\frac{1}{8(\lambda+\mu)^{2}}\left(-4(\lambda+\mu) g \lambda^{2}\left(\left(f_{2 y}+f_{1 x}\right)+6 \lambda \mu\left(\xi-\mathrm{u}_{2} x+\mathrm{u}_{1} y\right)+4 \mu^{2}\left(\xi-\mathrm{u}_{2} x+\mathrm{u}_{1} y\right)\right)\right. \\
& +\lambda^{2} \mu\left(f_{1 y}^{2}+2 f_{2 y}^{2}+2 f_{1 x}^{2}+f_{2 x}^{2}+2 f_{1 y} f_{2 x}+4 \mathrm{u}_{3}^{2} \phi_{y}^{2}+4 \mathrm{u}_{3}^{2} \phi_{x}^{2}+8 \mathrm{u}_{3}^{2} x \phi_{y}-8 \mathrm{u}_{3}^{2} y \phi_{x}+12 \xi^{2}\right. \\
& \left.+12 \mathrm{u}_{2}^{2} x^{2}+4 \mathrm{u}_{3}^{2} x^{2}-24 \xi \mathrm{u}_{2} x+24 \mathrm{u}_{1} y\left(\xi-\mathrm{u}_{2} x\right)+12 \mathrm{u}_{1}^{2} y^{2}+4 \mathrm{u}_{3}^{2} y^{2}\right)+\lambda^{3}\left(f_{2 y}+f_{1 x}\right)^{2} \\
& +4(\lambda+\mu)^{2}(\lambda+2 \mu) g^{2}+4 \lambda \mu^{2}\left(2 \mathrm{u}_{3}^{2} \phi_{y}^{2}+2 \mathrm{u}_{3}^{2} \phi_{x}^{2}+4 \mathrm{u}_{3}^{2} x \phi_{y}-4 \mathrm{u}_{3}^{2} y \phi_{x}+5 \xi^{2}+5 \mathrm{u}_{2}^{2} x^{2}+2 \mathrm{u}_{3}^{2} x^{2}\right. \\
& \left.-10 \xi \mathbf{u}_{2} x+10 \mathbf{u}_{1} y\left(\xi-\mathrm{u}_{2} x\right)+5 \mathbf{u}_{1}^{2} y^{2}+2 \mathbf{u}_{3}^{2} y^{2}\right)+4 \mu^{3}\left(\mathrm{u}_{3}^{2} \phi_{y}^{2}+\mathrm{u}_{3}^{2} \phi_{x}^{2}+2 \mathrm{u}_{3}^{2} x \phi_{y}-2 \mathbf{u}_{3}^{2} y \phi_{x}+2 \xi^{2}\right. \\
& \left.\left.+2 \mathrm{u}_{2}^{2} x^{2}+\mathrm{u}_{3}^{2} x^{2}-4 \xi \mathrm{u}_{2} x+4 \mathrm{u}_{1} y\left(\xi-\mathrm{u}_{2} x\right)+2 \mathrm{u}_{1}^{2} y^{2}+\mathrm{u}_{3}^{2} y^{2}\right)\right),
\end{aligned}
$$

and we can now integrate this expression over the cross section. This integrand is a function of the variables $\phi, f_{1}, f_{2}$ and their derivatives. However, an important realization is that the terms involving $f_{1}$ and $f_{2}$ in $V_{2}$ do not contain any dependence on the strains. Therefore, we can write:

$$
\mathcal{E}=\mathcal{E}_{\text {morphorod }}\left[\xi, \mathrm{u}_{1}, \mathrm{u}_{2}, \mathrm{u}_{3} ; g\right]+\mathcal{E}_{\text {reactive }}[g]
$$

where $\mathcal{E}_{\text {reactive }}$ is an energy term induced by the incremental growth that does not vary with the deformation of the central axis of the rod. Therefore, it is not necessary to integrate the $\mathcal{E}_{\text {reactive }}$ component in order to compute the properties of the equivalent Kirchhoff elastic rod (though for completeness this is included below).

To evaluate $\mathcal{E}$, we transform the integral by using integration by parts to transform nonlinear terms, the equations and boundary conditions that $\phi, f_{1}, f_{2}$ satisfy, and apply standard calculus identities to simplify the final result. Expressed in unscaled variables, the energy $\mathcal{E}_{\text {morphorod }}$ has the structure of a Kirchhoff elastic rod:

$$
\mathcal{E}_{\text {morphorod }}=\frac{1}{2} \int_{0}^{L}\left(K_{0}(\zeta-1)^{2}+K_{1}\left(\mathrm{u}_{1}-\hat{\mathrm{u}}_{1}\right)^{2}+K_{2}\left(\mathrm{u}_{2}-\hat{\mathrm{u}}_{2}\right)^{2}+K_{3} \mathrm{u}_{3}^{2}\right) \mathrm{d} Z,
$$

where we recover the usual quantities appearing in the classical rod theory

$$
\begin{aligned}
& K_{0}=\int_{\mathcal{S}} E \mathrm{~d} X \mathrm{~d} Y, \quad K_{1}=\int_{\mathcal{S}} E Y^{2} \mathrm{~d} X \mathrm{~d} Y, \quad K_{2}=\int_{\mathcal{S}} E X^{2} \mathrm{~d} X \mathrm{~d} Y, \\
& K_{3}=\int_{\mathcal{S}} \mu\left(Y^{2}+X^{2}\right) \mathrm{d} X \mathrm{~d} Y+\int_{\mathcal{S}} \mu\left(X \Phi_{Y}-Y \Phi_{X}\right) \mathrm{d} X \mathrm{~d} Y .
\end{aligned}
$$

Here $\Phi$ is the unscaled warping function, i.e. a solution of

$$
\begin{aligned}
& \Delta \Phi=0, \quad(X, Y) \in \mathcal{S} \\
& \mathbf{n} \cdot\left(\Phi_{X}, \Phi_{Y}\right)=\mathbf{n} .(Y,-X), \quad(X, Y) \in \partial \mathcal{S} .
\end{aligned}
$$


In addition, we define

$$
H_{1}=\int_{\mathcal{S}} E Y G(X, Y) \mathrm{d} X \mathrm{~d} Y, \quad H_{2}=\int_{\mathcal{S}} E X G(X, Y) \mathrm{d} X \mathrm{~d} Y
$$

where $G(X, Y)=\varepsilon g(X / \varepsilon, Y / \varepsilon)$. Then the unstressed curvatures are

$$
\hat{\mathrm{u}}_{1}=\frac{H_{1}}{K_{1}}, \quad \hat{\mathrm{u}}_{2}=-\frac{H_{2}}{K_{2}}, \quad \hat{\mathrm{u}}_{3}=0 .
$$

Finally, the reactive energy term is

$$
\begin{aligned}
\mathcal{E}_{\text {reactive }}=\frac{1}{2}\left(-K_{1} H_{1}-K_{2} H_{2}\right. & +\int_{\mathcal{S}} \frac{\nu-1}{2 \nu^{2}+\nu-1} E G^{2} \mathrm{~d} X \mathrm{~d} Y \\
& \left.+\int_{\mathcal{S}} \frac{\varepsilon^{2} E \nu^{2} G}{2 \nu^{2}+\nu-1}\left(f_{2 Y}\left(\frac{X}{\varepsilon}, \frac{Y}{\varepsilon}\right)+f_{1 X}\left(\frac{X}{\varepsilon}, \frac{Y}{\varepsilon}\right)\right) \mathrm{d} X \mathrm{~d} Y\right),
\end{aligned}
$$

though as stated, since it is independent of the strains, the reactive energy term does not contribute to the specification of the rod shape during deformation and can be ignored in transforming the grown structure to a $1 \mathrm{D}$ setting. We note that the form of the curvatures are similar to the results in $[20,21]$.

\subsubsection{A linear growth gradient}

If we assume a linear growth gradient of the form $g(x, y)=\gamma_{1} x+\gamma_{2} y$, then $G(X, Y)=\gamma_{1} X+\gamma_{2} Y$ and from (60) and (61), we have simply that

$$
\hat{\mathrm{u}}_{1}=\gamma_{2}, \quad \hat{\mathrm{u}}_{2}=-\gamma_{1} .
$$

We conclude that the amplitude of a linear growth gradient in the section gives the intrinsic curvature for all cross-sectional shapes of isotropic compressible materials.

\subsubsection{Generating intrinsic torsion}

At a first glance, it does not appear that the form of the curvatures allows for the generation of intrinsic torsion. However, an important observation is that all of the calculations above still carry through if the incremental growth field is a function of $Z$, so long as the rate of change in the $Z$-direction is $\mathcal{O}(1)$ or lower. Using this fact, a natural way to develop both curvature and torsion is for the growth gradient to twist along the axis. Consider, for instance, the growth field

$$
\mathbf{G}_{1}=\operatorname{diag}(0,0, \gamma(X \cos (\varphi Z)+Y \sin (\varphi Z))) .
$$

In this case, following the ideas above, (61) gives

$$
\hat{\mathrm{u}}_{1}=\gamma \sin (\omega Z), \quad \hat{\mathrm{u}}_{2}=-\gamma \cos (\omega Z) .
$$

To see that this form does in fact produce torsion, we note a fundamental relationship between torsion, which is a property of the centerline, and twist, a property describing rotation of the material frame (described further in Appendix A). The latter can be characterised by a function $\varphi(S)$ that describes the angle between the normal vector to the centerline and the vector $\mathbf{d}_{1}$. In general, curvature $\kappa$, twist $\varphi$, and torsion $\tau$ are related to the curvature vector by

$$
\mathbf{u}=\left(\kappa \sin \varphi, \kappa \cos \varphi, \tau+\frac{\partial \varphi}{\partial S}\right)
$$


In the case of the curvatures (65) generated by (64), we have $\hat{\mathbf{u}}_{3}=0$, hence

$$
\hat{\kappa}=\sqrt{\hat{\mathrm{u}}_{1}^{2}+\hat{\mathrm{u}}_{2}^{2}}=\gamma, \quad \hat{\tau}=-\frac{\partial \hat{\varphi}}{\partial S}=-\partial_{S}\left(\arctan \left(\hat{\mathrm{u}}_{1} / \hat{\mathrm{u}}_{2}\right)\right)=\omega .
$$

That is, a linearly rotating growth gradient produces a helix as illustrated in Fig. 2(b).

There are other possible mechanisms to generate torsion. One is to have a growth field creating residual shear in the section. This is obtained by having a growth tensor with non-diagonal components coupling the axial direction with any direction in the section. The shear created is partially removed by twisting the rod around its axis. By itself, this is not enough to generate torsion, But, in the presence of curvature, such twisting creates torsion. Another possible mechanism is to have an anisotropic material. For instance a fiber-reinforced material with helical fiber families will naturally generate torsion during axial growth in the presence of curvature. We also note in [22] a general formula for rods with prestrain oscillating on the lengthscale of the rod's thickness is given.

\subsubsection{Constant growth}

We consider the simple but important case of constant axial growth in a finite number $N$ of domains $\mathcal{S}_{i}$ such that $\mathcal{S}=\bigcup_{i=1}^{N} \mathcal{S}_{i}$ (see [33] for a similar construction). We denote by $\gamma_{i}$ the axial growth in each domain, so that

$$
\mathbf{G}_{1, i}=\operatorname{diag}\left(0,0, \varepsilon \gamma_{i}\right)
$$

Following the procedure above, we can formulate the energy as a sum of integrals over each of the domains. Defining $\boldsymbol{\alpha}_{i}$ as the deformation within the $i$ th section generates a series of Euler-Lagrange equations identical to (28)-(30); the only coupling between sub-domain appears in the boundary conditions. Indeed, the natural boundary condition only applies on the outside edge $\partial \mathcal{S}$, while a matching condition is needed on each interior boundary. However, recall the structure of the solutions (44)-(46): the geometry (and boundary conditions), as well as the growth, are contained in the functions $f_{1}, f_{2}$, which do not impact the properties of the rod. Moreover, while the warping function $\phi$ does depend on the geometry, it does not depend on the growth, hence this can be computed by consideration only of the external geometry. Therefore, the formulas (56)-(61) are still valid in the case of piecewise axial growth.

In particular, if we define the centroid of each domain (in unscaled variables) by $\left(X_{i}, Y_{i}\right)$, as measured from the centroid of the full cross-section, so that

$$
\int_{\mathcal{S}_{i}}\left(X-X_{i}\right) d X d Y=\int_{\mathcal{S}_{i}}\left(Y-Y_{i}\right) d X d Y=0
$$

then we obtain the intrinsic curvature components (see Eq. (60)) as sums of the first moments of area of the sub-sections weighted by growth:

$$
\begin{gathered}
H_{1}=\int_{\mathcal{S}} E Y G(X, Y) d X d Y=\sum_{i=1}^{N} \int_{\mathcal{S}_{i}} E_{i} Y \varepsilon \gamma_{i} d X d Y=\varepsilon \sum_{i=1}^{N} \gamma_{i} E_{i} Y_{i} A_{i}, \\
H_{2}=\int_{\mathcal{S}} E X G(X, Y) d X d Y=\sum_{i=1}^{N} \int_{\mathcal{S}_{i}} E_{i} X \varepsilon \gamma_{i} d X d Y=\varepsilon \sum_{i=1}^{N} \gamma_{i} E_{i} X_{i} A_{i},
\end{gathered}
$$

where $A_{i}$ is the area and $E_{i}$ the Young's modulus of the $i$ th domain. The intrinsic curvatures are then given by the ratio

$$
\hat{\mathrm{u}}_{1}=\frac{H_{1}}{K_{1}}, \quad \hat{\mathrm{u}}_{2}=-\frac{H_{2}}{K_{2}}
$$


where

$$
K_{1}=\int_{\mathcal{S}} E Y^{2} d X d Y=\sum_{i=1}^{N} \int_{\mathcal{S}_{i}} E_{i} Y^{2} d X d Y
$$

is a second moment of area, and similarly for $K_{2}$.

An example is given in Fig. 3. We take a simple scenario of six circular domains $\mathcal{S}_{i}, i \in$ $\{1,2, \ldots, 6\}$, inside a bounding circular domain $\mathcal{S}_{7}$. Constant axial growth is imposed in each domain as illustrated in Fig. 3(b), where a negative value indicates a contraction of that region. The curvature comes as a weighted average over the regions, and the resulting structure is plotted in Fig. 3(c). If either the orientation of the regions, or the growth, varies in the $Z$-direction, then growth can generate torsion. The former is demonstrated in Fig. 3(d). Here we have rotated the domains along the $Z$ axis in the pre-grown configuration, and the same piecewise growth produces a structure with curvature and torsion.

(a)

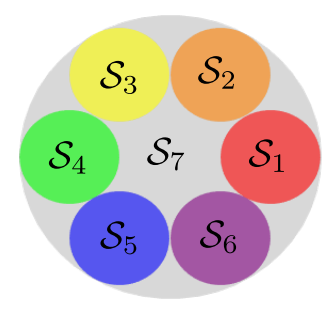

(c)

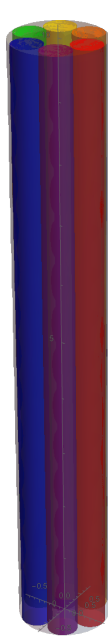

(b)

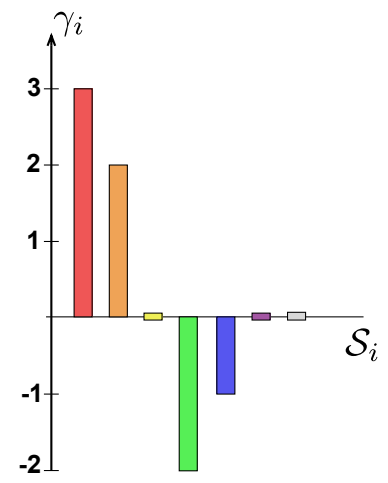

(d)

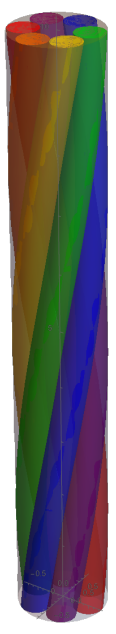

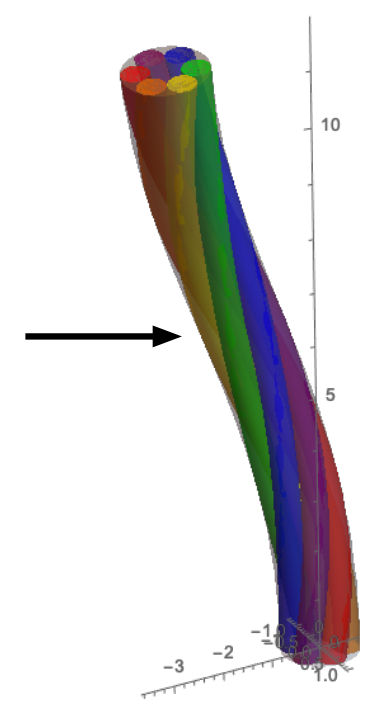

Figure 3: Constant axial growth in discrete domains. The domains illustrated in (a) are subjected to constant axial growth shown histographically in (b). (c) If the domains are uniform in the $Z$-direction, the growth generates curvature. (d) If the domains twist in the $Z$-direction, the same growth field produces torsion. Here $\mathcal{S}_{7}$ has radius $0.75, \mathcal{S}_{1}-\mathcal{S}_{6}$ have radius 0.25 , the total length $L=10$ in (c) and $L=12$ in (d), $\varepsilon=0.1$, and in (d) the domain rotates with rate 0.35 about the long axis. 


\section{Generating curvature with finite growth}

We now turn to the more difficult case of computing the rod parameters when finite growth is included, that is when $\mathbf{G}_{0}$ is not the identity. In the incompatible case, finite growth will generate residual stresses that will contribute to the rod's mechanics (see Section 4.2). In the compatible case, finite growth creates a new tubular body without stresses and the problem is trivial in the sense that one can define a new reference configuration and the previous discussion applies. However, this problem is still interesting as it allows us to include arbitrary uniform longitudinal growth, uniform twist, and uniform change of radius, and we solve this problem first.

\subsection{Compatible finite growth}

In cylindrical polar coordinates, we consider a deformation from $\left\{R_{0}, \Theta_{0}, Z_{0}\right\}$ to $\{R, \Theta, Z\}$. The most general growth deformation is an inflation, extension, and twisting of the structure, for which we have the map

$$
\begin{aligned}
R & =\lambda_{1} R_{0}, \\
\Theta & =\frac{\lambda_{2}}{\lambda_{1}} \Theta_{0}+\lambda_{4} Z_{0}, \\
Z & =\lambda_{3} Z_{0},
\end{aligned}
$$

where $\lambda_{1,2,3,4}$ are constant. The corresponding growth gradient in cylindrical to cylindrical coordinates

$$
\mathbf{G}_{0}=G_{0, \alpha \beta} \mathbf{e}_{\alpha} \otimes \mathbf{e}_{\beta}, \quad \alpha \in\{R, \Theta, Z\}, \quad \beta \in\left\{R_{0}, \Theta_{0}, Z_{0}\right\}
$$

is

$$
\mathbf{G}_{0}=\left[\begin{array}{ccc}
\lambda_{1} & 0 & 0 \\
0 & \lambda_{2} & \lambda_{4} R \\
0 & 0 & \lambda_{3}
\end{array}\right] .
$$

Following the deformation, the radius is stretched by a factor $\lambda_{1}$, the rod has extended axially by factor $\lambda_{3}$, and a twist of the cross-sections has been induced, with twisting rate $\lambda_{4}$. If this growth were combined with a generic incremental axial growth, we would compute the same exact curvatures as in Section 3.2, but the stiffnesses would be scaled by a factor of $\lambda_{1}^{4}$ in the case of bending and torsional stiffness and $\lambda_{1}^{2}$ in the case of extensional stiffness, due to the change in reference geometry. Also of interest here is that if an incremental axial growth gradient were defined in the initial configuration, e.g. $\mathbf{G}_{1}=\operatorname{diag}\left(1,1,1+\varepsilon \gamma R_{0} \cos \Theta_{0}\right)$, then since the finite growth induces a twist, the combination of twist and curvature would generate a torsion component $\hat{\tau}=\lambda_{4}$, similar to the calculation of (67) above. Compare this with the equivalent incremental growth field defined in the grown configuration, $\mathbf{G}_{1}=\operatorname{diag}(1,1,1+\varepsilon \gamma R \cos \Theta)$, which would not produce any torsion. In the latter case growth may be thought of as an axial gradient that is uniform along the $Z$ axis, imposed on a structure which has undergone already a growth remodelling step that twisted material points, but which is nevertheless stress-free.

\subsection{Incompatible finite growth}

Next, we consider the more complex scenario of combining incremental growth with incompatible finite growth. The idea is illustrated schematically in Fig. 4. Conceptually, we imagine that a cylindrical body undergoes an incompatible finite growth step $\mathbf{G}_{0}$, followed by an elastic response $\mathbf{A}_{0}$. In this step residual stress is induced but curvature is not; e.g. the growth field $\mathbf{G}_{0}$ could consist of radially symmetric inhomogeneous growth. The finite growth and elastic response maps the initial stress-free state $\mathcal{B}_{0}$ to a state $\mathcal{B}_{1}$ with non-zero Cauchy stress $\mathbf{T}$. The finite deformation 
uses no linearization, i.e. the full finite elasticity problem must be solved; this could be done by solving $\operatorname{div} \mathbf{T}=\mathbf{0}$ with stress-free boundary conditions in the standard way (see e.g. [34]); though we shall show that such a solution emerges naturally by simply following the steps we have outlined.

From the residually stressed but still cylindrical state $\mathcal{B}_{1}$, we then impose an incremental growth field $\mathbf{G}_{1}$ of the form considered thus far in this paper, which maps the body to $\mathcal{B}_{2}$, a configuration with non-zero intrinsic curvature. For instance, $\mathbf{G}_{1}$ could consist of axial growth with a crosssectional gradient. If the configuration $\mathcal{B}_{1}$ were stress-free, then we have already shown that if the body in $\mathcal{B}_{2}$ were approximated as an elastic rod, its intrinsic curvature would exactly match the growth gradient, and its stiffnesses would entirely be determined by the dimensions of configuration $\mathcal{B}_{1}$. But $\mathcal{B}_{1}$ is not stress free, and so the fundamental question is to determine if and how curvatures and stiffnesses are altered by the residual stress.

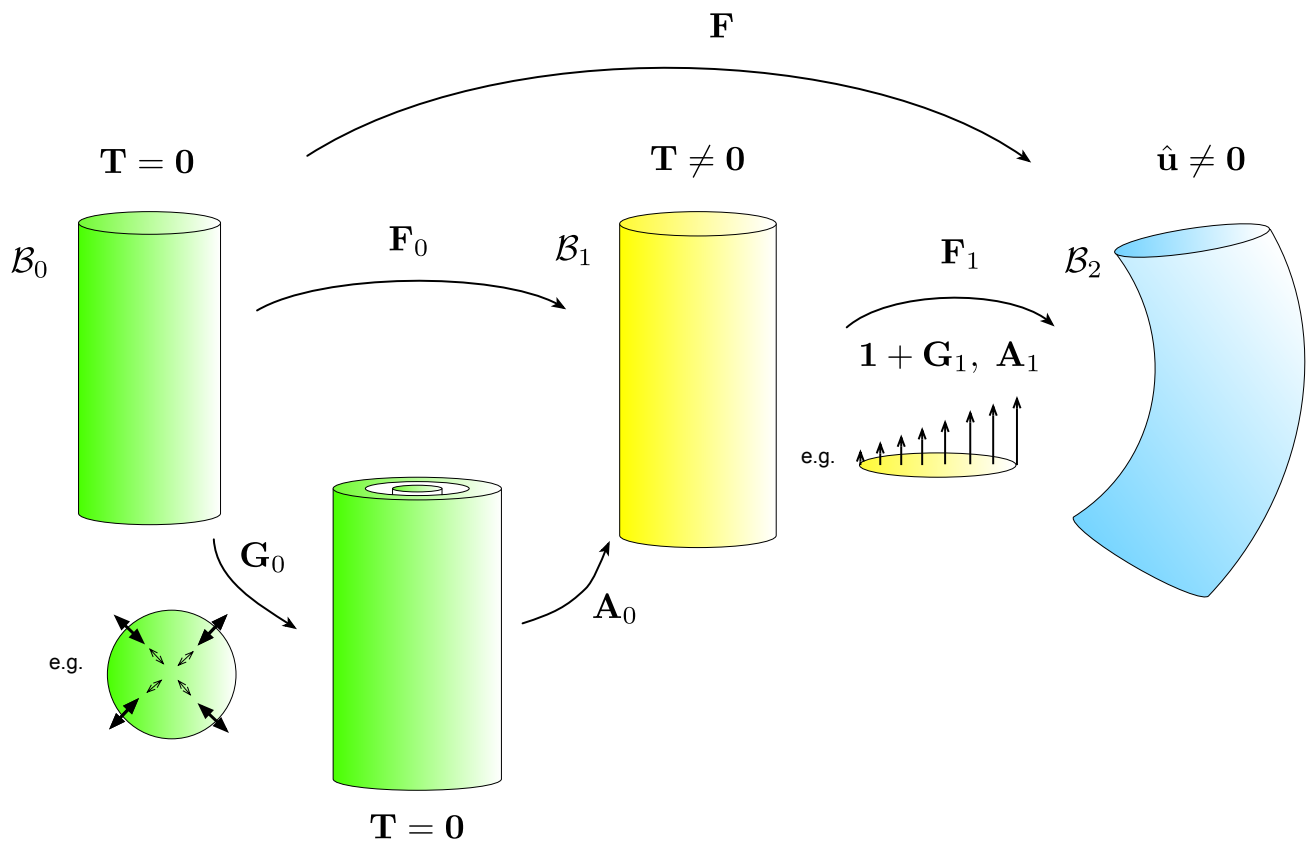

Figure 4: A cylindrical rod undergoes finite growth followed by curvature-inducing incremental growth. The finite growth preserves the cylindrical shape but induces residual stress, so that after an initial deformation $\mathbf{F}_{0}=\mathbf{A}_{0} \mathbf{G}_{0}$, the Cauchy stress $\mathbf{T} \neq \mathbf{0}$. An example would be a radially symmetric growth field that is a function of radius, e.g. the outside grows more than the inside. In this stressed but still cylindrical state, a non-uniform incremental axial growth $\mathbf{1}+\mathbf{G}_{1}$ is applied, which induces curvature.

Since the finite growth problem needs to be analytically tractable to make any progress, we take the cross sections to be circular with initial radius $R=\varepsilon$ in $\mathcal{B}_{0}$ and assume moreover that the material is incompressible, isotropic, and with neo-Hookean constitutive law. It is convenient to describe the configuration $\mathcal{B}_{0}$ by cylindrical polar coordinates $\{\hat{R}, \Theta, \hat{Z}\}^{1}$, and, similarly, the configuration $\mathcal{B}_{1}$ is described by $\{r, \theta, z\}$. We write the growth tensor in cylindrical to cylindrical variables:

$$
\mathbf{G}=\left(\mathbf{1}+\mathbf{G}_{1}\right) \mathbf{G}_{0}=G_{\alpha \beta} \mathbf{e}_{\alpha} \otimes \mathbf{e}_{\beta}, \quad \alpha \in\{r, \theta, z\}, \beta \in\{\hat{R}, \Theta, \hat{Z}\} .
$$

While the ideas outlined below can be generalised to any finite growth step that preserves the cylindrical geometry, for illustration we restrict our attention to a particular form of the finite

\footnotetext{
${ }^{1}$ The hat notation is introduced here to enable scaled variables below to be without hat.
} 
growth tensor:

$$
\mathbf{G}_{0}=\operatorname{diag}(\gamma(\hat{R}), \gamma(\hat{R}), 1) .
$$

For the incremental growth, we consider for now the generic axial form

$$
\mathbf{G}_{1}=\operatorname{diag}(0,0, \varepsilon g(\hat{R}, \Theta)),
$$

satisfying

$$
\int_{0}^{2 \pi} \int_{0}^{\varepsilon} g(\hat{R}, \Theta) \hat{R} \mathrm{~d} \hat{R} \mathrm{~d} \Theta=0 .
$$

While it may be more natural to define $\mathbf{G}_{1}$ in the variables of the deformed configuration $\mathcal{B}_{1}$ (and we shall do so below), for computational simplicity we work in $\hat{R}$, the variable corresponding to the initial configuration, and where needed we may utilise the one-to-one map $r(\hat{R})$ from $\mathcal{B}_{0}$ to $\mathcal{B}_{1}$.

The deformation from $\mathcal{B}_{0}$ to $\mathcal{B}_{2}$ may be thought of as a composition of maps, as pictured in Fig. 4, i.e. $\mathbf{F}=\mathbf{F}_{1} \mathbf{F}_{0}=\mathbf{A}_{1}\left(\mathbf{1}+\mathbf{G}_{1}\right) \mathbf{A}_{0} \mathbf{G}_{0}$. Following the ideas in [35], since $\mathbf{A}_{0}, \mathbf{G}_{0}$, and $\mathbf{G}_{1}$ are diagonal, these tensors commute and the deformation is equivalent to $\mathbf{F}=\mathbf{A}_{1} \mathbf{A}_{0}\left(\mathbf{1}+\mathbf{G}_{1}\right) \mathbf{G}_{0}$, i.e. this particular deformation can be simplified and treated with a single growth step $\mathbf{G}=\left(\mathbf{1}+\mathbf{G}_{1}\right) \mathbf{G}_{0}$ from the stress-free configuration $\mathcal{B}_{0}$, followed by elastic response tensor $\mathbf{A}=\mathbf{A}_{1} \mathbf{A}_{0}$.

Using the scaling (7), we have $\hat{R}=\varepsilon R$, and the deformation gradient $\mathbf{F}=\mathbf{A G}=F_{i \alpha} \mathbf{d}_{i} \otimes \mathbf{e}_{\alpha}, i \in$ $\{1,2,3\}, \alpha \in\{R, \Theta, Z\}$ is given by

$$
\mathbf{F}=\left[\begin{array}{ccc}
\alpha_{1 R} & \frac{1}{R} \alpha_{1 \Theta} & \lambda \varepsilon(1+\varepsilon \xi)\left(\mathrm{u}_{2} \alpha_{3}-\mathrm{u}_{3} \alpha_{2}\right) \\
\alpha_{2 R} & \frac{1}{R} \alpha_{2 \Theta} & \lambda \varepsilon(1+\varepsilon \xi)\left(\mathrm{u}_{3} \alpha_{1}-\mathrm{u}_{1} \alpha_{3}\right) \\
\alpha_{3 R} & \frac{1}{R} \alpha_{3 \Theta} & \lambda(1+\varepsilon \xi)\left(1+\varepsilon\left(\mathrm{u}_{1} \alpha_{2}-\mathrm{u}_{2} \alpha_{1}\right)\right)
\end{array}\right]
$$

Aside from being written explicitly in cylindrical polar coordinates, the only difference in the form of $\mathbf{F}$ at this stage is the presence of $\lambda$, which is the axial extension due to the finite growth.

The neo-Hookean energy is given by

$$
W(\mathbf{A})=\frac{\mu}{2}\left(\operatorname{tr}\left(\mathbf{A} \mathbf{A}^{\mathrm{T}}\right)-3\right),
$$

and, as before, we define the auxiliary energy density

$$
V=(W(\mathbf{A})-p(J-1)) \operatorname{det} \mathbf{G},
$$

where $J=\operatorname{det} \mathbf{A}$.

We proceed as before, expanding the inner variables $\alpha$ and $p$, with the auxiliary energy density taking the form

$$
V\left(\mathbf{F G}^{-1}, \mathbf{G}\right)=V_{0}\left(\alpha_{1}^{(0)}, \alpha_{2}^{(0)}, \alpha_{3}^{(0)}, p^{(0)}, \lambda\right)+\varepsilon^{2} V_{2}\left(\alpha_{1}^{(1)}, \alpha_{2}^{(1)}, \alpha_{3}^{(1)}, p^{(1)}\right) .
$$

where we have omitted the linear terms in $\varepsilon$ as they vanish in the variational process as well as higher order terms. The Euler-Lagrange equations are given by

$$
\begin{aligned}
& \frac{\partial}{\partial R} \frac{\partial V_{i}}{\partial \alpha_{j R}^{(k)}}+\frac{1}{R} \frac{\partial V_{i}}{\partial \alpha_{j R}^{(k)}}+\frac{\partial}{\partial \Theta} \frac{\partial V_{i}}{\partial \alpha_{j \Theta}^{(k)}}-\frac{\partial V_{i}}{\partial \alpha_{j \Theta}^{(k)}}=0, \quad j=1,2,3, k=0,1, \\
& \frac{\partial}{\partial R} \frac{\partial V_{i}}{\partial p_{R}^{(k)}}+\frac{1}{R} \frac{\partial V_{i}}{\partial p_{R}^{(k)}}+\frac{\partial}{\partial \Theta} \frac{\partial V_{i}}{\partial p_{\Theta}^{(k)}}-\frac{\partial V_{i}}{\partial p_{\Theta}^{(k)}}=0, \quad k=0,1 .
\end{aligned}
$$


with natural boundary conditions

$$
\begin{aligned}
& \left.\frac{\partial V_{i}}{\partial \alpha_{j R}^{(k)}}\right|_{R=1}=0 \quad j=1,2,3, k=0,1, \\
& \left.\frac{\partial V_{i}}{\partial p_{R}^{(k)}}\right|_{R=1}=0 \quad k=0,1 .
\end{aligned}
$$

We solve these equations order by order. At lowest order, the Euler-Lagrange equations are satisfied by

$$
\boldsymbol{\alpha}^{(0)}=r(R)(\cos \Theta, \sin \Theta, 0), \quad p^{(0)}=P(R),
$$

with $r(R)$ and $P(R)$ solving

$$
\begin{aligned}
& r^{\prime}=\frac{R \gamma^{2}}{\lambda r}, \\
& P^{\prime}=-\frac{\mu\left(-2 \lambda R^{3} r^{2} \gamma \gamma^{\prime}-2 \lambda R^{2} r^{2} \gamma^{2}+\lambda^{2} r^{4}+R^{4} \gamma^{4}\right)}{\lambda^{3} R r^{4}},
\end{aligned}
$$

and boundary conditions

$$
r(0)=0, \quad P(1)=\frac{\mu \gamma_{1}^{2}}{a^{2} \lambda^{2}} .
$$

where $\gamma_{1}=\gamma(1)$ and $a=r(1)$ is the rod's radius after finite growth. The constant $\lambda$ is not prescribed by this variational problem, but since the configuration $\mathcal{B}_{1}$ is assumed to be unloaded, the extra condition appears from the consideration of null traction on the sectional face of the cylinder as described in [16, p. 480]. Note that the boundary-value problem for $r(R), P(R)$ is equivalent to that obtained by considering $\mathbf{G}=\mathbf{G}_{0}$ only and solving $\operatorname{div} \mathbf{T}=\mathbf{0}$ with $\mathbf{T}=\mathbf{A}(\partial W / \partial \mathbf{A})-p \mathbf{1}$ and $\mathbf{T} \mathbf{n}=\mathbf{0}$ on the boundary, with normal vector $\mathbf{n}$.

At $\mathcal{O}\left(\varepsilon^{0}\right)$ we have the same solution as $(89)$

$$
\boldsymbol{\alpha}^{(0)}=x \mathbf{d}_{1}+y \mathbf{d}_{2}
$$

but in the deformed configuration $\mathcal{B}_{1}$, so that $x=r(R) \cos \Theta, y=r(R) \sin \Theta$.

At $\mathcal{O}(\varepsilon)$, the Euler-Lagrange equations are again automatically satisfied, and we turn to the more difficult problem of determining the solution to second-order.

At $\mathcal{O}\left(\varepsilon^{2}\right)$, the key to making progress is to make the correct ansatz on the form of the $\alpha_{i}^{(1)}$ and $p^{(1)}$. Based on the form of solution at leading order (89) and the symmetry of the problem, we use the following ansatz:

$$
\begin{aligned}
& \alpha_{1}^{(1)}=-\frac{1}{2} r \xi \cos \Theta-\mathrm{u}_{1} q_{1} \sin 2 \Theta+\mathrm{u}_{2}\left(q_{3} \cos 2 \Theta+\int^{R} q_{2}(\rho) \mathrm{d} \rho\right)+f_{1}, \\
& \alpha_{2}^{(1)}=-\frac{1}{2} r \xi \sin \Theta+\mathrm{u}_{1}\left(q_{1} \cos 2 \Theta+\int^{R} q_{4}(\rho) \mathrm{d} \rho\right)+\mathrm{u}_{2} q_{3} \sin 2 \Theta+f_{2}, \\
& \alpha_{3}^{(1)}=\mathrm{u}_{3} \phi(R, \Theta), \\
& p_{1}^{(1)}=-\xi q_{5}+\mathrm{u}_{1} q_{6} \sin \Theta+\mathrm{u}_{2} q_{7} \cos \Theta,
\end{aligned}
$$

where $q_{i}, i=1, \ldots, 7$ are functions of $R$; these must satisfy a linear system of first order ODEs, which is given in Appendix $\mathrm{C}$, and $f_{1,2}$, as before, are functions that only enter in the reactive part of the energy and are not needed to compute the moduli and intrinsic curvature. The function $\phi(R, \Theta)$ is the same warping function that appears in the linear problem; but for cylindrical geometry we have simply $\phi \equiv 0$. We note that this ansatz yields an exact solution but it only provides a critical point of the functional and not necessarily the global minimizer. 


\subsubsection{Example: linear growth}

To demonstrate the theory and the effect of finite growth on the rod's parameters, we consider the following form for the finite growth:

$$
\mathbf{G}_{0}=\operatorname{diag}(\gamma(R), \gamma(R), 1) \text {, with } \gamma(R)=1+c R,
$$

and prescribe incremental growth

$$
\mathbf{G}_{1}=\operatorname{diag}(0,0, \varepsilon g(R, \Theta)) \text {, with } g(R, \Theta)=r(R) \cos \Theta .
$$

The incremental growth is axial with a linear gradient in the deformed configuration $\mathcal{B}_{1}$, since $x=r \cos \Theta$ in $\mathcal{B}_{1}$. If $c=0$, the finite growth is turned off, so $r=R$, and thus based on the results of Section 3.2 we expect the incremental growth to produce a curvature $\hat{\mathrm{u}}_{2}=-1$, as well as standard stiffnesses for a circular rod.

We compute the rod's parameters as follows. For each value of $c$, we first determine the finite deformation $r(R), P(R)$ by solving the boundary value problem (90)-(92) via a numerical shooting method. In doing this we must also determine the axial stretch $\lambda$ by prescribing a zero traction condition on the face of the cylinder. Using the constitutive relation for the $Z$ component of the Cauchy stress, $t_{Z Z}=\lambda(\partial W / \partial \lambda)-p$, for a neo-Hookean strain-energy density $W$ this condition can be written as

$$
\int_{0}^{1}\left(\mu \lambda^{2}-P(R)\right) r(R) r^{\prime}(R) \mathrm{d} R=0 .
$$

The solution $\{r(R), P(R), \lambda\}$ is fed into the linear system for the $q_{i}(R)$, which is again solved via numerical shooting. The result is a numerical representation of the $\alpha_{i}^{(1)}$ and $p^{(1)}$. Once these have been determined, the second order energy density takes the form

$V_{2}=A_{1}(R, \Theta) \xi+A_{2}(R, \Theta) \xi^{2}+B_{1}(R, \Theta) \mathbf{u}_{1}+B_{2}(R, \Theta) \mathbf{u}_{1}^{2}+C_{1}(R, \Theta) \mathbf{u}_{2}+C_{2}(R, \Theta) \mathbf{u}_{2}^{2}+D_{2}(R, \Theta)$.

The stiffnesses and curvatures are found by integrating over the cross-section and completing the square. In particular, the intrinsic curvature and bending stiffness about $\mathbf{d}_{2}$ are given respectively by

$$
\hat{\mathrm{u}}_{2}=-\int_{0}^{2 \pi} \int_{0}^{1} \frac{C_{1}(R, \Theta)}{2 C_{2}(R, \Theta)} R \mathrm{~d} R \mathrm{~d} \Theta, \quad K_{2}=\int_{0}^{2 \pi} \int_{0}^{1} C_{2}(R, \Theta) R \mathrm{~d} R \mathrm{~d} \Theta
$$

and these integrals can be computed numerically as part of the shooting procedure. We note that the equations for the $q_{i}$ decouple for each strain component, which can be used to simplify the computation. Thus for instance the 3 functions that multiply $\mathrm{u}_{2}\left(q_{2}, q_{3}, q_{7}\right)$ satisfy a coupled system of 3 equations that is independent of the other $q_{i}$. Determining $\hat{\mathbf{u}}_{2}$ only requires computing these 3 functions. By the symmetry of the finite growth and the form of the incremental growth, $K_{1}=K_{2}$ and $\hat{\mathrm{u}}_{1}=0$, though these can also be verified computationally following similar calculations as above.

In Fig. 5 we plot the (negative of the) intrinsic curvature (Fig. 5(a)) and bending stiffness (Fig. 5(b)) as a function of the growth parameter $c$. At $c=0$. we find $\hat{\mathrm{u}}_{2}=-1$, and the bending stiffness $K_{2}=E \pi / 4$, both as expected. For $c>0$, the intrinsic curvature is higher (in absolute value), and is lower for $c<0$. This confirms that enhanced or diminished curvature may be attained through residual stress. In terms of the stiffness, for any $c \neq 0$ the dimensions of the cylinder in configuration $\mathcal{B}_{1}$ are changed due to the finite growth. Hence, to see the impact of the residual stress, we compare the stiffness $K_{2}$ as given by (102) to the stiffness of a circular rod with the same dimensions and zero residual stress. That is, we compare $K_{2}$ to the quantity $E \pi a^{4} / 4$, where $a=r(1)$ is the radius in the deformed state $\mathcal{B}_{1}$. The latter is plotted as the dashed red 

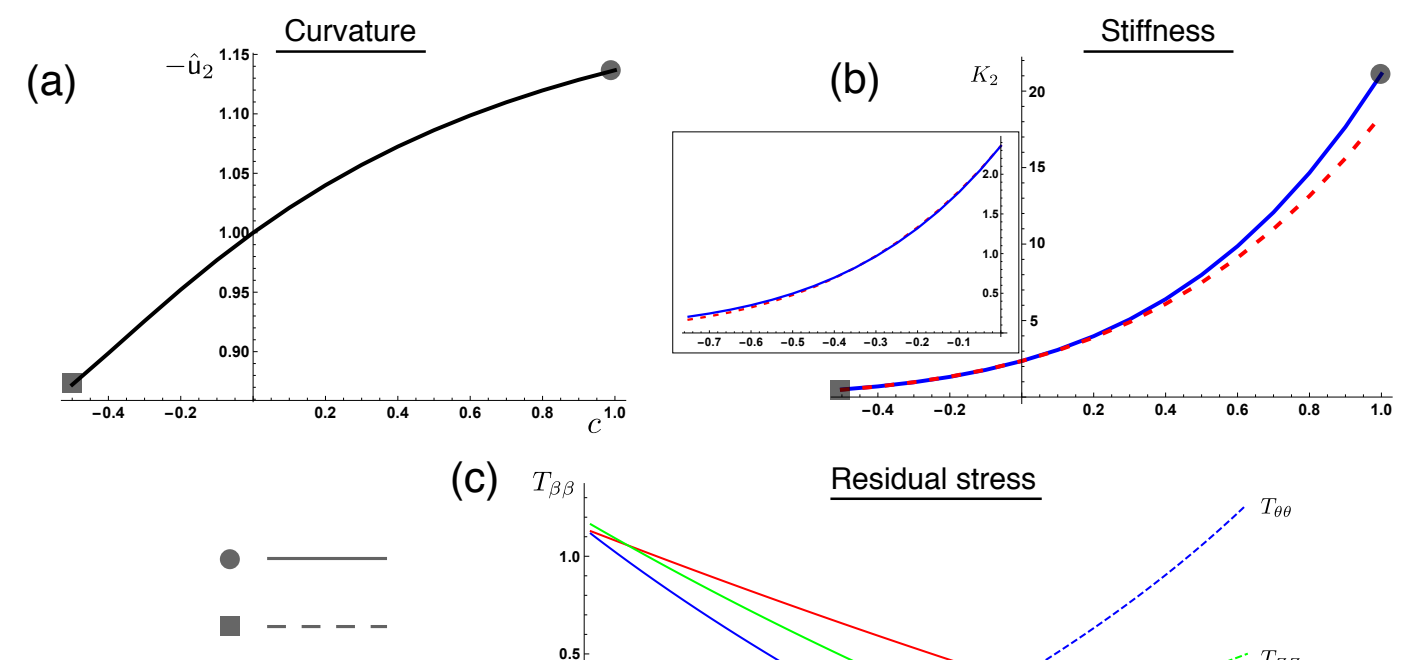

(c)

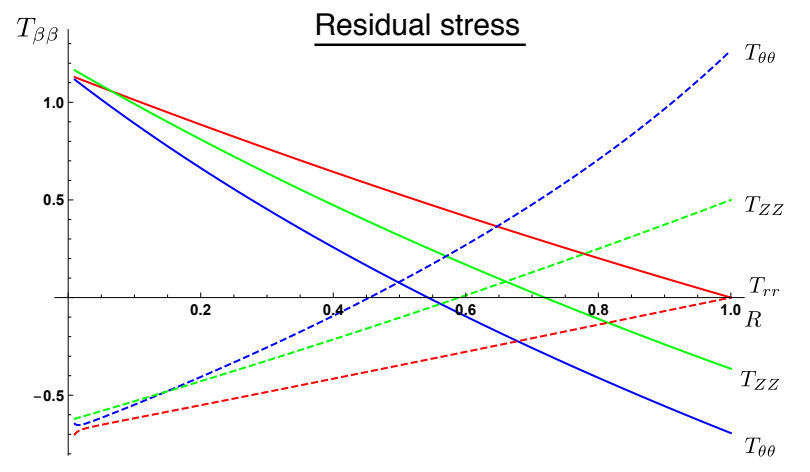

Figure 5: Finite inhomogeneous radial growth $\gamma=1+c R$ is combined with incremental axial growth with a constant gradient. The curvature (a) and bending stiffness (b) are plotted as functions of the finite growth gradient $c$. In (b), the dashed red curve is the stiffness that would be obtained based on the change in geometry alone due to the finite growth, i.e. if there were no residual stress. In (c), the stress profiles following the finite growth are plotted for the marked points $c=1$ and $c=-0.5$.

line in Fig. 5(b), and we see that the rod with finite growth has increased stiffness compared to an equivalent rod with no residual stress. The inset shows a zoom-in of this plot in the region $c<0$, and extended to $c=-0.75$; perhaps surprisingly the stiffness here is also increased due to the residual stress, albeit by a very small amount.

Finally, in Fig. 5(c) we plot the stress components in configuration $\mathcal{B}_{1}$ at the marked points $c=1$ and $c=-0.5$. These two points show a qualitatively opposite behaviour: at $c=1$ the outside of the cylinder is in both compressive hoop stress and axial stress; while at $c=-0.5$ the outside of the cylinder is in tensile hoop stress and axial stress; vice versa at the center of the cylinder. It is evident that this qualitative difference in residual stress profiles is driving the qualitatively different responses to an imposed linear gradient in axial growth, demonstrating the significant and non-trivial impact of residual stress on curvature generation.

\section{Conclusion}

In this paper we have developed a framework for linking the microscopic growth of filamentary structures to the macroscopic properties. At the microscopic scale, we model growth by a tensor field that locally defines the increase or decrease of mass in each direction at each point in a 3-dimensional elastic material. From there, we mapped to an equivalent macroscopic structure, treated as an elastic rod characterised by 4 stiffness moduli and an intrinsic curvature vector. Our theory requires only two assumptions: (i) that the 3 -dimensional structure has a small aspect ratio 
between the cross-sectional dimensions and its length, and (ii) that any curvature-inducing growth is of the same order as the aspect ratio. From these two assumptions we have outlined a stepby-step procedure for transforming from growth tensor to rod properties, and have demonstrated the utility via a series of examples. Our analysis was first restricted to the case of incremental growth only. Here we obtained a generic formula for the intrinsic curvatures in terms of integrals involving the axial growth function; this uncovered the simple yet instructive result that a linear growth gradient will produce an intrinsic curvature with matching gradient, for any cross-sectional geometry.

Similar expressions for the curvature, without axial extension, have been obtained using Gamma convergence [20, 21, 22] or asymptotic methods [36]. Here, our main objective was to derive such results within the framework of morphoelasticity and asymptotic methods, and in a fashion that could easily be applied to specific problems in biological growth and/or extended to have additional finite growth. Indeed, with incremental growth combined with finite growth, we showed how the same framework still applies. While the computations are complicated by the finite growth part, in principle the problem can be solved, and the method was illustrated via an example. Here we found the important result that residual stress, induced by the finite growth step, can alter both the stiffness and the intrinsic curvature of the averaged macroscopic filament.

While our primary motivation was towards biological applications, the ideas and framework we have outlined may also have significant relevance in engineering design, for instance in the burgeoning field of soft robotics [37], or in morphing structures [38], where residual stress can be built into the fabric of a material in order to achieve a design aim. In such contexts a robust map between material properties at the microstructure and the material response at the macroscale is critical. In terms of growing filamentary structures in biology, one obvious example, noted in the introduction, is in tropic plant growth, in which parts of a plant develop curvature and/or torsion in response to external stimuli such as gravity or light. This phenomenon is inherently a multiscale process, whereby the global orientation of the plant in its external environment drives cellular activities, which in turn generate differential growth at the tissue level, which then in turn generates curvature and changes the global properties and orientation. While most models of tropism focus on one scale, a full treatment of tropic growth could be achieved by linking a model of cellular activities to the continuum micro to macro transformation we have described. A less obvious but still relevant phenomenon is the incredible dexterity of the elephant's trunk. This is achieved through differential contraction of over 40,000 muscles. In an idealized view, the contraction of any set of muscles could be modeled using the same tensorial field $\mathbf{G}$ with the condition that no mass is added $(\operatorname{det} \mathbf{G}=1)$.

Any of the applications noted above would require significant efforts to be modelled appropriately. Nevertheless, at a fundamental level, these problems involve microscopic changes of a filamentary structure creating a global change in properties that can be captured by the methods outlined here, and viewed in this sense the realm for interesting potential future studies is vast.

Acknowledgments- This work was supported by the Engineering and Physical Sciences Research Council grant EP/R020205/1 to Alain Goriely.

\section{A A brief review of rod theory}

Here we briefly recall the basic elements of rod geometry. A $\operatorname{rod}$ is a space curve $\mathbf{r}(S) \in \mathbb{R}^{3}$, known as the centerline, equipped with two additional unit orthonormal vector fields $\left(\mathbf{d}_{1}(S), \mathbf{d}_{2}(S)\right)$ representing the orientation of a cross section at $S$. The general frame is obtained by defining $\mathbf{d}_{3}(S)=\mathbf{d}_{1}(S) \times \mathbf{d}_{2}(S)$ and we note that $\left\{\mathbf{d}_{\mathbf{1}}, \mathbf{d}_{\mathbf{2}}, \mathbf{d}_{\mathbf{3}}\right\}$ forms a right-handed orthonormal basis. The components of a vector $\mathbf{a}=\mathrm{a}_{1} \mathbf{d}_{1}+\mathrm{a}_{2} \mathbf{d}_{2}+\mathrm{a}_{3} \mathbf{d}_{3}$ in the local basis are denoted by $\mathbf{a}=\left(\mathrm{a}_{1}, \mathrm{a}_{2}, \mathrm{a}_{3}\right)$. 
We note that $|\mathbf{a}|=|\mathbf{a}|$.

We choose the material parameter $S$ to be the arc length in a stress-free reference configuration, $\widehat{\mathbf{r}}$ of the space curve [39]. In the current configuration, we have

$$
\frac{\partial \mathbf{r}}{\partial S}=\mathbf{v}
$$

where $\mathbf{v}$ is the stretch vector, with $\|\mathbf{v}\|>0$. A complete kinematic description of the frame is given by:

$$
\frac{\partial \mathbf{d}_{i}}{\partial S}=\mathbf{U} \times \mathbf{d}_{i}, \quad i=1,2,3,
$$

where $\mathbf{U}$ is the material Darboux vector. The first two components $\left(\mathrm{U}_{1}, \mathrm{U}_{2}\right)$ of the Darboux vector are associated with the Frenet curvature while $U_{3}$ represents twisting, that is the rotation of the basis (not the curve) around the $\mathbf{d}_{3}$ vector. It contains both information on the Frenet torsion $\tau$ of the centerline and on the rotation of the cross section for increasing values of $S$.

If the rod is unshearable but extensible, then $\mathrm{v}_{1}=\mathrm{v}_{2}=0, \mathrm{v}_{3}=\zeta$ and we can introduce the current form of the Darboux vector as $\mathbf{U}=\zeta \mathbf{u}$. The components of this Darboux vector gives the actual curvatures in the current deformation (rather than the curvatures with respect to the material frame). We have then

$$
\frac{\partial \mathbf{d}_{i}}{\partial S}=\zeta \mathbf{u} \times \mathbf{d}_{i}, \quad i=1,2,3,
$$

which is the form used in this paper.

In particular, if the rod is assumed to be inextensible and unshearable, then $v_{1}=v_{2}=0, v_{3}=1$ and $\mathbf{d}_{3}$ is along the tangent. In this case, the Darboux vector is related to the usual notion of Frenet curvature and torsion $\kappa$ and $\tau$ are the by

$$
\begin{aligned}
& \cot \varphi=\frac{\mathrm{u}_{2}}{\mathrm{u}_{1}}, \\
& \kappa=\sqrt{\mathrm{u}_{1}^{2}+\mathrm{u}_{2}^{2}}, \\
& \tau=\mathrm{u}_{3}+\frac{\mathrm{u}_{2}^{\prime} \mathrm{u}_{1}-\mathrm{u}_{1}^{\prime} \mathrm{u}_{2}}{\mathrm{u}_{1}^{2}+\mathrm{u}_{2}^{2}} .
\end{aligned}
$$

where $\varphi$ is the angle between the normal and the vector $\mathbf{d}_{1}$. The quantity $\frac{\partial \varphi}{\partial S}$, the excess twist, represents the rotation of the local basis with respect to the Frenet frame as the arc length increases. We note that torsion and curvature are properties of the Frenet frame, while bend and twist are properties of the material frame attached to the rod.

The contact force $\mathbf{n}(S)$ exerted on the back side of $\mathcal{S}(S)$ by the previous sections is called the resultant force at $S$, and we define $\mathbf{m}(S)$ as being the resultant couple acting on a section at $S$ due to the adjacent segment with larger material coordinates.

A general model for the elastic response of the rod is governed by a strain-energy density (per unit material length)

$$
w=w(\mathbf{y}, \mathbf{z})=w(\mathbf{u}-\hat{\mathbf{u}}, \mathbf{v}-\hat{\mathbf{v}}),
$$

where $\hat{\mathbf{u}}, \hat{\mathbf{v}}$ are the strains in the unloaded configuration, i.e. for which $\mathbf{n}=\mathbf{m}=\mathbf{0}$. Then the constitutive relations for the resultant moment and force in the local basis are given by

$$
\begin{aligned}
\mathbf{m} & =\partial_{\mathbf{y}} w(\mathbf{u}-\hat{\mathbf{u}}, \mathbf{v}-\hat{\mathbf{v}})=\mathbf{f}(\mathbf{u}-\hat{\mathbf{u}}, \mathbf{v}-\hat{\mathbf{v}}) \\
\mathbf{n}=\partial_{\mathbf{z}} w(\mathbf{u}-\hat{\mathbf{u}}, \mathbf{v}-\hat{\mathbf{v}}) & =\mathbf{g}(\mathbf{u}-\hat{\mathbf{u}}, \mathbf{v}-\hat{\mathbf{v}}) .
\end{aligned}
$$


The case that is of interest for the analysis in this paper is an extensible but unshearable rod with a quadratic elastic energy $w$. In this case, we have $\mathrm{v}_{1}=\mathrm{v}_{2}=0$, and $w$ can be written

$$
w=\mathbf{y}^{\top} \mathbf{K} \mathbf{y}+K_{0}(\zeta-1)^{2},
$$

where the final term is the energy of an axial elastic stretch $\zeta \equiv \mathrm{v}_{3} \equiv \partial s / \partial S$. The constitutive relations for the moment in the local basis components are

$$
\mathbf{m}=\mathbf{K}(\mathbf{u}-\hat{\mathbf{u}}), \quad \mathbf{K}=\left(\begin{array}{ccc}
K_{1} & K_{12} & K_{13} \\
K_{12} & K_{2} & K_{23} \\
K_{13} & K_{23} & K_{3}
\end{array}\right), K_{1} \geq K_{2} .
$$

The condition $K_{1} \geq K_{2}$ corresponds to the choice of labeling of the vectors $\mathbf{d}_{1}$ and $\mathbf{d}_{2}$. If, in a basis $K_{1}<K_{2}$ then a proper re-labeling of these vectors leads to $K_{1} \geq K_{2}$. Also, note that, in general, we can choose the vector $\mathbf{d}_{1}$ so that, without loss of generality, we have $K_{12}=K_{21}=0$. The theory so far is an exact theory of one-dimensional systems as explained in $[40,16]$. This theory is not sufficient to specify the values of the stiffness coefficients $K_{i j}$ in terms of the geometric and material properties of the underlying elastic material. They can be obtained by considering the deformations of an elastic cylinder as done originally by Kirchhoff [41], by using a centre-manifold approach [42] or by Gamma-convergence [17].

In the simplest and most widely used case, the constitutive equations have the form

$$
\begin{aligned}
& \mathbf{m}=K_{1}\left(\mathrm{u}_{1}-\hat{\mathrm{u}}_{1}\right) \mathbf{d}_{1}+K_{2}\left(\mathrm{u}_{2}-\hat{\mathrm{u}}_{2}\right) \mathbf{d}_{2}+K_{3}\left(\mathrm{u}_{3}-\hat{\mathrm{u}}_{3}\right) \mathbf{d}_{3}, \\
& \mathrm{n}_{3}=K_{0}(\zeta-1) .
\end{aligned}
$$

In this case, the Kirchhoff theory tells us that the stifnesses are

$$
K_{0}=E A, \quad K_{1}=E I_{1}, \quad K_{2}=E I_{2}, \quad K_{3}=\mu J
$$

where $E$ is the Young's modulus, $\mu$ the second Lamé parameter and $J, I_{1,2}$ depend on the crosssectional shape (see main text).

Note that since $S$ is not the arc length, the Darboux vector $\mathbf{u}$ is scaled by an elastic stretch factor $\zeta$ so that

$$
\mathbf{u}=\zeta\left(\kappa \sin \varphi, \kappa \cos \varphi, \tau+\frac{\partial \varphi}{\partial s}\right)
$$

However, the intrinsic Darboux vector $\hat{\mathbf{u}}$ is given by the intrinsic geometric curvatures in the reference configuration; it is not scaled by $\zeta$ since it is a material property of the rod in the reference configuration. The elastic energy of the rod is then

$$
\mathcal{E}_{\text {rod }}=\frac{1}{2} \int_{0}^{L}\left(K_{0}(\zeta-1)^{2}+K_{1}\left(\mathrm{u}_{1}-\hat{\mathrm{u}}_{1}\right)^{2}+K_{2}\left(\mathrm{u}_{2}-\hat{\mathrm{u}}_{2}\right)^{2}+K_{3}\left(\mathrm{u}_{3}-\hat{\mathrm{u}}_{3}\right)^{2}\right) \mathrm{d} S .
$$

\section{B Solving the Euler-Lagrange equations}

We look for a formal solution of the linear PDE for a given function $g$

$$
\begin{aligned}
& \mu f_{1 y y}+(2 \mu+\lambda) f_{1 x x}+\mu f_{2 x y}+\lambda f_{2 x y}-2(\mu+\lambda) g_{x}=0 \\
& (\lambda+\mu) f_{1 x y}+(2 \mu+\lambda) f_{2 y y}+\mu f_{2 x x}-2(\mu+\lambda) g_{y}=0
\end{aligned}
$$


Assuming that $g$ is $C^{\infty}$ in a finite number $N$ of domains $\mathcal{S}_{i}$ such that $\mathcal{S}=\bigcup_{i=1}^{N} \mathcal{S}_{i}$, a formal solution in each domain $\mathcal{S}_{i} \ni(x, y)$ is given by

$$
\begin{aligned}
f_{1}= & \frac{1}{2 \mu+\lambda} \sum_{n=0}^{\infty}(-)^{n+1}((2 n-1) \lambda+(2 n-2) \mu) \int \mathrm{d}^{2 n+1} x\left(\frac{\partial^{2 n}}{\partial y^{2 n}} g(x, y)\right)+ \\
& \frac{1}{2 \mu+\lambda} \sum_{n=0}^{\infty}(-)^{n+1}((2 n+1) \lambda+(2 n+2) \mu) \int \mathrm{d}^{2 n+2} y\left(\frac{\partial^{2 n+1}}{\partial x^{2 n+1}} g(x, y)\right) \\
f_{2}= & \frac{1}{2 \mu+\lambda} \sum_{n=0}^{\infty}(-)^{n+1}((2 n-1) \lambda+(2 n-2) \mu) \int \mathrm{d}^{2 n+1} y\left(\frac{\partial^{2 n}}{\partial x^{2 n}} g(x, y)\right)+ \\
& \frac{1}{2 \mu+\lambda} \sum_{n=0}^{\infty}(-)^{n+1}((2 n+1) \lambda+(2 n+2) \mu) \int \mathrm{d}^{2 n+2} x\left(\frac{\partial^{2 n+1}}{\partial y^{2 n+1}} g(x, y)\right),
\end{aligned}
$$

This solution is exact if $g$ is a polynomial in two variables. The case of a constant growth with linear gradient $g(x, y)=\gamma_{0}+\gamma_{1} x+\gamma_{2} y$ on a sub-domain is independent of the material parameters:

$$
f_{1}=\gamma x+\gamma_{2} x y+\frac{1}{2} \gamma_{1}\left(x^{2}-y^{2}\right), \quad f_{2}=\gamma y+\gamma_{1} x y-\frac{1}{2} \gamma_{2}\left(x^{2}-y^{2}\right) .
$$

\section{Solving the Euler-Lagrange equations with finite growth}

Given a function $\gamma=\gamma(R)$, the radial deformation $r(R)$ is a solution of (90). Then the crosssectional variables $q_{i}$ are solutions of the linear system

$$
\begin{gathered}
q_{1}^{\prime}=\frac{\lambda q_{4} r^{2}-2 R q_{1} \gamma^{2}+R r^{2} \gamma^{2}}{\lambda r^{2}} \\
q_{2}^{\prime}=-\frac{R^{2} \gamma^{4}\left(r^{2}-5 q_{3}\right)}{\lambda^{2} r^{4}}+\frac{R \gamma\left(r^{2}-4 q_{3}\right) \gamma^{\prime}}{\lambda r^{2}}- \\
\frac{\gamma^{2}\left(-\lambda q_{7} r-2 \mu R q_{2}+8 \mu q_{3}+\left(\lambda^{3}-2\right) \mu r^{2}\right)}{2 \lambda \mu r^{2}}-\frac{R q_{2}+q_{3}}{R^{2}} \\
q_{3}^{\prime}=\frac{-\lambda q_{2} r^{2}-2 R q_{3} \gamma^{2}+R r^{2} \gamma^{2}}{\lambda r^{2}}, \\
\frac{R^{2} \gamma^{4}\left(r^{2}-5 q_{1}\right)}{\left.\lambda^{2} r^{4}-\frac{R \gamma\left(r^{2}-4 q_{1}\right) \gamma^{\prime}}{\lambda r^{2}}+2 \mu\left(4 q_{1}+R q_{4}\right)+\left(\lambda^{3}-2\right) \mu r^{2}\right)} \\
2 \lambda \mu r^{2} \\
q_{6}^{\prime}=\frac{q_{1}-R q_{4}}{\lambda^{3} R r^{5}}\left(16 \lambda \mu R^{3} q_{1} r^{2} \gamma \gamma^{\prime}+4 \lambda \mu R^{3} q_{4} r^{2} \gamma^{2}+\lambda^{2} R^{2} q_{6} r^{3} \gamma^{2}+16 \lambda \mu R^{2} q_{1} r^{2} \gamma^{2}-2 \lambda^{2} \mu R^{2} r^{4} q_{4}^{\prime}\right) \\
\frac{1}{\lambda^{3} R r^{5}}\left(4 \lambda^{2} \mu q_{1} r^{4}-2 \lambda^{2} \mu R q_{4} r^{4}-20 \mu R^{4} q_{1} \gamma^{4}+5 \mu R^{4} r^{2} \gamma^{4}-6 \lambda \mu R^{3} r^{4} \gamma \gamma^{\prime}-6 \lambda \mu R^{2} r^{4} \gamma^{2}+\lambda^{2} \mu r^{6}\right) \\
q_{5}^{\prime}=\frac{2 \lambda R^{3} r^{2} \gamma \gamma^{\prime}+2 \lambda R^{2} r^{2} \gamma^{2}-\lambda^{2} r^{4}-R^{4} \gamma^{4}}{\lambda^{3} R r^{4}},
\end{gathered}
$$




$$
\begin{aligned}
q_{7}^{\prime}= & \frac{1}{\lambda^{3} R r^{5}}\left(\mu \left(-R^{2} r^{2} \gamma\left(8 \lambda R q_{3} \gamma^{\prime}+\gamma\left(8 \lambda q_{3}-2 \lambda R q_{2}\right)+3 R^{2} \gamma^{3}\right)\right.\right. \\
& \left.\left.\quad+\lambda r^{4}\left(-2 \lambda q_{3}+4 R^{3} \gamma \gamma^{\prime}+\left(\lambda^{3}+4\right) R^{2} \gamma^{2}\right)\right)+\frac{1}{\lambda^{3} R r^{5}}\left(+10 R^{4} q_{3} \gamma^{4}-\lambda^{2} r^{6}\right)\right)
\end{aligned}
$$

\section{References}

[1] U Kutschera. Gravitropism of axial organs in multicellular plants. Adv. Space Res., 27(5):851860, 2001.

[2] R. Bastien, T. Bohr, B. Moulia, and S. Douady. Unifying model of shoot gravitropism reveals proprioception as a central feature of posture control in plants. Proc. Natl. Acad. Sci. USA, 110(2):755-760, 2013.

[3] OM O'Reilly and TN Tresierras. On the static equilibria of branched elastic rods. International journal of engineering science, 49(2):212-227, 2011.

[4] W. K. Silk. On the curving and twining of stems. Environ. Exper. Bot., 29:95-109, 1989.

[5] W. K. Silk. Growth rate patterns which maintain a helical tissue tube. J. Theor. Biol., 138(3):311-327, 1989.

[6] K. Schulgasser and A. Witztum. The mechanism of spiral grain formation in trees. Wood Sci. Technol., 41(2):133-156, 2006.

[7] A. Goriely and M. Tabor. Spontaneous rotational inversion in Phycomyces. Phys. Rev. Lett., 106(13):138103, 2011.

[8] A. Goriely and M. Tabor. Nonlinear dynamics of filaments I: Dynamical instabilities. Phys. D, 105:20-44, 1997.

[9] O. M. O'Reilly. Modeling Nonlinear Problems in the Mechanics of Strings and Rods: The Role of the Balance Laws. Springer New York, 2016.

[10] D E Moulton, T Lessinnes, and A Goriely. Morphoelastic rods Part I: A single growing elastic rod. J. Mech. Phys. Solids, 61(2):398-427, 2012.

[11] Th. Lessinnes, D. E. Moulton, and A. Goriely. Morphoelastic rods. Part II: Growing birods. J. Mech. Phys. Solids, 100:147-196, 2017.

[12] D. E. Moulton, A. Goriely, and R. Chirat. Mechanical growth and morphogenesis of seashells. J. Theor. Biol., 311:69-79, 2012.

[13] R. Chirat, D. E. Moulton, and A. Goriely. Mechanical basis of morphogenesis and convergent evolution of spiny seashells. Proc. Natl. Acad. Sci. USA, 110(15):6015-6020, 2013.

[14] R. E. Goldstein and A. Goriely. Dynamic buckling of morphoelastic filaments. Phys. Rev. E, 74:010901, 2006.

[15] A. Goriely and D. E. Moulton. Morphoelasticity - a theory of elastic growth. In Oxford University Press, editor, New Trends in the Physics and Mechanics of Biological Systems, 2010. 
[16] A. Goriely. The Mathematics and Mechanics of Biological Growth. Springer Verlag, New York, 2017.

[17] Maria Giovanna Mora and Stefan Müller. Derivation of the nonlinear bending-torsion theory for inextensible rods by $\Gamma$-convergence. Calculus of Variations and Partial Differential Equations, 18(3):287-305, 2003.

[18] Alain Cimetière, Giuseppe Geymonat, Herve Le Dret, Annie Raoult, and Zvonimir Tutek. Asymptotic theory and analysis for displacements and stress distribution in nonlinear elastic straight slender rods. Journal of elasticity, 19(2):111-161, 1988.

[19] Jacqueline Sanchez-Hubert and Evarisre Sanchez Palencia. Statics of curved rods on account of torsion and flexion. European Journal of Mechanics-A/Solids, 18(3):365-390, 1999.

[20] Robert V Kohn and Ethan O'Brien. On the bending and twisting of rods with misfit. $J$. Elasticity, (in Press), 2017.

[21] Marco Cicalese, Matthias Ruf, and Francesco Solombrino. On global and local minimizers of prestrained thin elastic rods. Calculus of Variations and Partial Differential Equations, 56(4):115, 2017.

[22] Robert Bauer, Stefan Neukamm, and Mathias Schäffner. Derivation of a homogenized bendingtorsion theory for rods with micro-heterogeneous prestrain. arXiv preprint arXiv:1903.08290, 2019.

[23] Raz Kupferman and Jake P Solomon. A Riemannian approach to reduced plate, shell, and rod theories. Journal of Functional Analysis, 266(5):2989-3039, 2014.

[24] Marta Lewicka and Mohammad Reza Pakzad. Scaling laws for non-euclidean plates and the w 2, 2 isometric immersions of riemannian metrics. ESAIM: Control, Optimisation and Calculus of Variations, 17(4):1158-1173, 2011.

[25] S. M. Klisch and A. Hoger. Volumetric growth of thermoelastic materials and mixtures. Math. Mech. Solids, 8:377 - 402, 2003.

[26] Vlado A Lubarda. Elastoplasticity theory. CRC press, 2001.

[27] A Goriely. Five ways to model active processes in elastic solids: Active forces, active stresses, active strains, active fibers, and active metrics. Mechanics Research Communications, 93:7579, 2018.

[28] A. Yavari and A. Goriely. Nonlinear elastic inclusions in isotropic solids. Proc. Roy. Soc. Lond. A, 469(2160):20130415, 2013.

[29] V. A. Lubarda. Constitutive theories based on the multiplicative decomposition of deformation gradient: Thermoelasticity, elastoplasticity, and biomechanics. Appl. Mech. Rev., 57:95-108, 2004 .

[30] M. Ben Amar and A. Goriely. Growth and instability in elastic tissues. J. Mech. Phys. Solids, 53:2284-2319, 2005.

[31] S. S. Antman and R. S. Marlow. Material constraints, Lagrange multipliers, and compatibility. applications to rod and shell theories. Arch. Rat. Mech., 116:257-299, 1991. 
[32] E. K. Rodriguez, A. Hoger, and A. McCulloch. Stress-dependent finite growth in soft elastic tissue. J. Biomech., 27:455-467, 1994.

[33] Sébastien Turcaud, Anders Thorin, Yves Bréchet, Peter Fratzl, and John WC Dunlop. Twisters: an analogy of bilayers for twisting. Journal of the Mechanics and Physics of Solids, 134:103742, 2020 .

[34] Martine Ben Amar and Alain Goriely. Growth and instability in elastic tissues. Journal of the Mechanics and Physics of Solids, 53(10):2284-2319, 2005.

[35] Alain Goriely and Martine Ben Amar. On the definition and modeling of incremental, cumulative, and continuous growth laws in morphoelasticity. Biomechanics and modeling in mechanobiology, 6(5):289-296, 2007.

[36] Claire Lestringant and Basile Audoly. Asymptotically exact strain-gradient models for nonlinear slender elastic structures: a systematic derivation method. Journal of the Mechanics and Physics of Solids, page 103730, 2019.

[37] Panagiotis Polygerinos, Nikolaus Correll, Stephen A Morin, Bobak Mosadegh, Cagdas D Onal, Kirstin Petersen, Matteo Cianchetti, Michael T Tolley, and Robert F Shepherd. Soft Robotics: Review of Fluid-Driven Intrinsically Soft Devices; Manufacturing, Sensing, Control, and Applications in Human-Robot Interaction? Advanced Engineering Materials, 521(5):e201700016, May 2017.

[38] C K Khoo, F Salim, and J Burry. Designing architectural morphing skins with elastic modular systems. International Journal of .., 2011.

[39] N. Chouaieb, A. Goriely, and J. H. Maddocks. Helices. Proc. Natl. Acad. Sci. USA, 103(25):9398-9403, 2006.

[40] S. S. Antman. Nonlinear problems of elasticity. Springer New York, 2005.

[41] G. Kirchhoff. Vorlesungen über mathematische Physik, Mechanik, volume 28. B. G. Teubner, Leipzig, 1876.

[42] Alexander Mielke. On saint-venant's problem for an elastic strip. Proceedings of the Royal Society of Edinburgh Section A: Mathematics, 110(1-2):161-181, 1988. 